\title{
La prova nel processo penale ${ }^{1}$
}

\author{
Evidence in criminal procedure \\ A prova no processo penal
}

Paolo Ferrua

Ordinario di Procedura penale Università degli Studi di Torino/Itália

paolo.ferrua@unito.it

http://orcid.org/0000-0002-7459-6762

\begin{abstract}
AвSTRACT: Lo scritto analizza le tre componenti dell'operazione probatoria: le premesse probatorie o prove in senso stretto, con particolare riguardo alla distinzione tra dichiarazioni di prova e prove critico- indiziarie; le proposizioni da provare, principali o incidentali, finali o intermedie; l'atto del provare, connotato dalla regola dell'oltre ogni ragionevole dubbio. Mentre i primi due termini variano in funzione del contesto processuale, il terzo resta indefettibile, essendo contraddittorio ritenere 'provata' una qualsiasi proposizione allorché vi sia un ragionevole motivo per dubitarne. Quanto alla ripartizione degli oneri probatori, determinante è la struttura della fattispecie, sostanziale o processuale. In base a questa è possibile individuare, per ogni alternativa decisoria, il termine 'marcato', che veicola la proposizione da provare, e l'opposto termine 'consequenziale', che deriva dal mancato raggiungimento della prova: ad esempio, rispetto al tema principale del processo, termine 'marcato' è la condanna, termine 'consequenziale' il proscioglimento.

PAROLE ChIAVE: prova; ragionevole dubbio; processo penale.
\end{abstract}

ABSTRACT: This paper analyzes the three components of the evidence operation: proof premises or evidence in the strict sense, with particular regard to the distinction between evidence declaration and critical/circumstantial evidence;

1 Lo scritto riproduce, con alcune varianti, parte dei capitoli III-IV del volume La prova nel processo penale. Vol. I, Struttura e procedimento. $2^{\circ}$ ed. Torino: Giappichelli, 2017. 
the propositions to be proved, principal or incidental, final or intermediate; the act of proving, connoted by the rule of beyond all reasonable doubt. While the first two terms vary according to the procedural context, the third remains indefectible, since it is incongruous to consider any proposition to be 'proven' while there is a reasonable reason to doubt it. With regard to the distribution of the burden of proof, the structure of the case and its legal qualification, whether substantial or procedural, are decisive. Therefore, it is possible to identify, for each decision alternative, the term 'marked', which conveys the proposition to be proved, and the opposite 'consequential' term, which derives from the failure to reach the proof: for example, with respect to the main object in trial, the term 'marked' is the conviction, the term 'consequential' the acquittal.

KEYWORDs: evidence; reasonable doubt; criminal procedure.

Resumo: Este artigo analisa os três elementos da operação probatória: $a$ premissa probatória ou prova em sentido estrito, com especial atenção à distinção entre declarações probatórias e prova crítica-indiciária; as proposições a serem provadas, principais ou incidentais, finais ou intermediárias; o ato de provar, definido pela regra de "além da dúvida razoável". Enquanto os dois primeiros variam de acordo com o contexto processual, o terceiro permanece inalterado, uma vez que é contraditório considerar qualquer proposição "comprovada" enquanto houver uma razão razoável para duvidar disso. No que diz respeito à distribuição do ônus da prova, é determinante a estrutura do tipo legal, seja substancial ou processual. Com base nisso, é possível identificar, para cada alternativa decisória, o elemento "marcado", que transmite a proposição a ser provada e o elemento "consequencial" oposto, derivado da falha na comprovação: por exemplo, em relação ao objeto principal em julgamento, o elemento "marcado" é a condenação, e o elemento "consequencial" é a absolvição.

Palavras-chave: prova; dúvida razoável; processo penal.

Sommario: 1. La prova come operazione a tre termini: premesse probatorie, proposizione da provare, atto del provare. - 2. Regole di esclusione e criteri di valutazione: due fenomeni da non confondere. - 3. Negazione passiva e negazione attiva: non presunzione di colpevolezza e presunzione di non colpevolezza (o di innocenza). - 4. Ammissibilità ed efficacia persuasiva della prova. - 5. Equivoci in tema di indizi. - 6. Premesse probatorie: prove dirette e prove indirette. - 7. Prove precostituite e prove costituite. - 8. Dichiarazioni di prova e prove critico-indiziarie. - 9. Criteri identificativi 
della prova dichiarativa. L'atto comunicativo. - 10. L'enunciato apofantico. - 11. Prove critico-indiziarie. - 12. Prove critico-indiziarie artificiali e naturali: la struttura complessa dell'intercettazione. - 13. "Sequenze" e "convergenze" probatorie. - 14. Proposizioni da provare. - 15. L'atto del provare e il ragionamento abduttivo. - 16. La regola dell'oltre ogni ragionevole dubbio: due modelli di prova. - 17. 'La prova oltre ogni ragionevole dubbio' tra regola e principio: i c.d. casi difficili. - 18. Il dubbio ... sulla 'ragionevolezza' del dubbio. - 19. L'oltre ogni ragionevole dubbio e il teorema di Bayes. - 20. Funzione pedagogica della regola di giudizio: povertà denotativa e ricchezza connotativa. - 21. Alternative decisorie: termine 'marcato' e 'termine' consequenziale. - 22. Onere della prova. - 23. L'oltre ogni ragionevole dubbio e il rischio dell'abuso nei provvedimenti cautelari. - 24. Presunzione di innocenza e prova oltre ogni ragionevole dubbio. Bibliografia.

\section{LA PROVA COME OPERAZIONE A TRE TERMINI: PREMESSE PROBATORIE, PROPOSIZIONE DA PROVARE, ATTO DEL PROVARE}

La prova, latamente intesa, è un'operazione volta a verificare, quindi ad accertare come vera o falsa una qualsiasi proposizione ${ }^{2}$. In essa si possono individuare tre componenti:

a) i mezzi potenzialmente idonei a provare, id est le prove in senso proprio che saranno formalizzate e descritte in altrettante proposizioni o premesse probatorie;

b) l'oggetto o il tema della prova, ossia la proposizione che si intende provare: la quale dovrà essere costituita da un enunciato apofantico, vale a dire vero o falso, dato che non avrebbe senso provare enunciati né veri né falsi, come comandi, promesse, consigli, ordini o, più in generale, tutti gli enunciati performativi;

c) il criterio o la regola di giudizio alla cui stregua si può ritenere raggiunta la prova e, quindi, effettuato con esito positivo il passaggio

2 Nell'etimo 'provare' significa riconoscere qualche cosa come buona; e le proposizioni oggetto di prova in tanto sono 'buone', in quanto siano 'vere'. 
dalle proposizioni probatorie alla proposizione da provare: la regola, già implicita nel concetto stesso di 'provare', è rappresentata dal canone dell'oltre ogni ragionevole dubbio ${ }^{3}$.

Prova, nel senso di premessa probatoria, è ogni dato che, legittimamente acquisito al processo, sia valutabile dal giudice in ordine a una determinata proposizione da provare. Non ha senso chiedersi se un elemento costituisca o no prova, senza avere contestualmente individuato la proposizione da provare ${ }^{4}$. Ciò che è prova in ordine al tema $x$, può non esserlo su quello $y$. Ad esempio, una dichiarazione raccolta unilateralmente nelle indagini preliminari è prova sul tema cautelare (i gravi indizi di colpevolezza o i pericula libertatis) o su quello della condotta illecita ex art. 500 comma 4 c.p.p., ma non lo è in sede dibattimentale sul tema della colpevolezza (salve le eccezioni contemplate dalla legge).

Premesse probatorie e proposizioni da provare sono i termini variabili del meccanismo probatorio, o meglio le prime variano in funzione delle seconde; le quali vanno attentamente individuate, perché, come si vedrà, non sempre la legge le specifica in modo trasparente. L'atto del provare è, invece, la componente invariabile della terna, perché, come vedremo, in qualsiasi contesto processuale è sempre connotato dal medesimo criterio: quali che siano le prove e quale che sia il tema probatorio, una proposizione può dirsi "provata" solo in quanto lo sia "oltre ogni ragionevole dubbio"; la permanenza di un dubbio ragionevole è incompatibile con l'esito positivo dell'operazione probatoria.

\section{Regole di ESCLUSIONE E CRITERI DI VALUTAZIONE: DUE FENOMENI DA NON CONFONDERE}

Collegata alla struttura triadica della prova è la distinzione tra regole di esclusione probatoria e criteri di valutazione. Le prime riguardano la prova come premessa probatoria; e - variamente espresse ora nella forma del divieto di ammissione ora del divieto di utilizzazione

3 Infra par. 16.

4 A meno, s'intende, che siano dati giuridicamente inesistenti per i quali è $a$ priori esclusa ogni rilevanza probatoria. 
- operano sempre in un momento antecedente a quello in cui interverrebbe la valutazione, proprio perché negano alla radice l'idoneità di un certo dato a fungere da valida premessa probatoria ${ }^{5}$.

La prova che sia stata acquisita, violando una regola di esclusione, è giuridicamente inesistente, tamquam non esset. A sanzionare il divieto probatorio non occorre una specifica previsione di nullità o di altro vizio processuale, che avrebbe anzi l'effetto di convertire l'inesistenza giuridica in un vizio meno grave come la nullità. L'irrilevanza processuale della prova non ammessa dalla legge è in re ipsa. Se la prova è vietata dalla legge, il giudice non ha il potere di assumerla; e l'atto, eventualmente compiuto in assenza del potere, cade nel vuoto. Un atto in tanto può produrre l'effetto cui è preordinato in quanto chi lo compie sia investito del relativo potere.

Che una prova inammissibile sia inutilizzabile è un'affermazione vera, ma tautologica, nella quale il predicato non aggiunge nulla al soggetto. Da questo punto di vista, in quanto riferito alle prove inammissibili, l'art. 191 c.p.p. è una disposizione pleonastica; o, meglio, si spiega come monito del legislatore a non cadere negli abusi di un passato dove, in forza del libero convincimento del giudice, si recuperavano al processo anche prove illegittimamente ammesse (incluse le testimonianze estorte).

Le regole di esclusione possono essere 'assolute' (come per gli scritti anonimi, per le dichiarazioni estorte, per le voci correnti nel pubblico, ecc.); o 'relative', quando operano solo in rapporto ad una determinata proposizione da provare (ad es., sul tema della colpevolezza) o nei confronti di certi imputati (ad es., coloro la cui difesa non abbia partecipato all'assunzione dell'atto).

Ben diversa la disciplina dei criteri legali di valutazione: a differenza delle regole di esclusione, implicano prove validamente costituite e riguardano il passaggio da queste alla proposizione da provare che viene in vario modo guidato dalla legge. Essendo spesso enunciati nella forma " $q$ vale come prova di $p$ solo in presenza di $r$ ", si può essere indotti a convertirli in una regola di esclusione del tipo " $q$, in assenza di $r$, è un dato

5 Per un approfondimento del tema cfr. M. DANIELE, Regole di esclusione e regole di valutazione della prova, Giappichelli, Torino, 2009. 
inutilizzabile": ad esempio, dire che una dichiarazione può valere come prova solo in presenza di elementi che ne confermano l'attendibilità così il criterio fissato dall'art. 192 comma 3 c.p.p. - non equivale forse a dire che, mancando tali elementi, la dichiarazione è inutilizzabile? $\mathrm{E}$ istintivo rispondere positivamente; ma è un errore, favorito ancora una volta dall'ambiguità del termine 'prova'. Il criterio legale non incide sulla valida costituzione della prova, intesa come dato valutabile dal giudice; influisce solo sul valore della prova sino ad annullarlo in assenza di certi requisiti. Nell'esempio di cui sopra, la dichiarazione del coimputato è 'prova' (si allude ad una potenzialità del dato legittimamente acquisito) e, come tale, dev'essere valutata dal giudice; ma, in forza della regola legale, è inidonea a 'provare' (il verbo indica un esito) il fatto dichiarato, in assenza di riscontri.

Insomma, una cosa è che un dato non possa essere valutato perché inutilizzabile (id est, non acquisibile come prova), sottratto a priori al convincimento giudiziale; un'altra che per effetto dei criteri legali il valore di una prova si riduca o addirittura scenda a zero rispetto a determinati esiti: la negazione che nel primo caso precede la valutazione, nel secondo la segue. È in sostanza l'elementare distinzione tra negazione passiva ("non valuto che ...") e negazione attiva ("valuto che non ...") rispetto alla proposizione affermativa ("valuto che ...") ${ }^{6}$. La regola di esclusione vieta la valutazione del giudice, il criterio la condiziona e la guida, sino a prefigurarne a volte l'esito negativo, come accade con le dichiarazioni del coimputato che, prive di riscontri, non provano il fatto dichiarato. In quest'ultimo caso il risultato non è, all'atto pratico, diverso da quello che deriverebbe da una regola di esclusione, perché la proposizione oggetto di prova non sarà provata; ma concettualmente la distinzione tra prova inutilizzabile e prova il cui valore è in certe circostanze nullo resta netta e rilevante a vari effetti. Ad esempio, ai fini del ricorso in cassazione, da esperire rispettivamente ai sensi della lett. c) e della lett. $e$ ) dell'art. 606 c.p.p.; o ai fini del diritto intertemporale, perché nel primo caso la legge applicabile è quella vigente al momento dell'acquisizione, nel secondo quella al momento della valutazione.

6 Per la rilevanza della distinzione sul terreno delle contestazioni ex art. 500 c.p.p., v. P. FerruA, Il "giusto processo", III ed., Zanichelli, Bologna, 2012, 144 s. 


\section{Negazione PASSIVA E NEGAZIONE ATTIVA: NON PRESUNZIONE DI COLPEVOLEZZA E PRESUNZIONE DI NON COLPEVOLEZZA (O DI INNOCENZA)}

La distinzione tra negazione passiva ("non valutare che") e negazione attiva ("valutare che non") è singolarmente trascurata, come si può notare a proposito del principio contenuto nell'art. 27 comma 2 Cost.: «L'imputato non è considerato colpevole sino alla condanna definitiva». Principio solo in parte assimilabile a quello espresso dall'art. 6 comma 2 CEDU («Ogni persona accusata di un reato è presunta innocente sino a quando la sua colpevolezza non sia stata legalmente accertata») ${ }^{7}$.

Nonostante si tratti di formule diverse, la 'non considerazione di colpevolezza' della Costituzione viene solitamente convertita nella 'presunzione di innocenza o di non colpevolezza' (innocente e non-colpevole sono termini sinonimi ${ }^{8}$ ), al punto che assai raramente la si cita nel suo tenore letterale. A tale esito concorrono, sicuramente, esigenze di semplificazione, di uniformità col testo della Convenzione europea e, storicamente, una comprensibile carica polemica verso il regime fascista che aveva definito la presunzione di innocenza «una stravaganza derivante da quei vieti concetti, germogliati dai principi della Rivoluzione francese, per cui si portano ai più esagerati e incoerenti eccessi le garanzie individuali»?.

Tuttavia, dal punto di vista testuale, l'equiparazione tra le due formule è un grave errore logico, alla cui origine sta la confusione tra la 'negazione attiva' e la 'negazione passiva'. La Costituzione, lungi dall'imporre di 'presumere' l'innocenza o il suo equivalente logico, la 'non colpevolezza', dice soltanto che «l'imputato non è considerato

7 C. Santoriello, Il vizio di motivazione tra esame di legittimità e giudizio di fatto, Utet, Torino, 2008, $52 \mathrm{s.}$

8 Anche etimologicamente, separando il prefisso dal radicale, in-nocente è colui che non nuoce. Sul bilanciamento tra presunzione di innocenza e anticipazione di effetti tipici della sanzione penale, v. M. CAIANiELlo, Il principio di proporzionalità nel procedimento penale, in Diritto penale contemporaneo, 18 giugno 2014; G. Illuminati, La presunzione d'innocenza, Zanichelli, Bologna, 1979; R. ORLANDI, Rito penale e salvaguardia dei galantuomini, in Criminalia, 2006, 303; P.P. PAulesu, La presunzione di non colpevolezza, Giappichelli, Torino, 2009.

9 Così il guardasigilli Alfredo Rocco nella Relazione al progetto preliminare del codice di proc. pen., in Lavori preparatori del codice penale e del codice di procedura penale, vol. VIII, Roma, 1929, 22. 
colpevole». La negazione (il 'non') è riferita al verbo ('considerato') e non al sostantivo ("colpevole").

Nel linguaggio comune spesso queste differenze sfuggono, ma c'è un netto divario tra dire che l'imputato 'è considerato non colpevole' (negazione attiva) o che 'non è considerato colpevole' (negazione passiva). Nel primo caso si predica un giudizio di 'non colpevolezza' del tutto equivalente a quello di innocenza; nel secondo si predica l'agnosticismo, l'astensione dal giudizio di colpevolezza. In tutti i verbi epistemici (credere, ritenere, provare, presumere, pensare, ecc.) la negazione attiva o passiva assume un significato ben diverso. Che poi nella vulgata si ignorino queste varianti e si preferisca parlare, in un caso come nell'altro, di presunzione di innocenza (o di non colpevolezza), è un dato di fatto che non scalfisce minimamente la rilevanza del discorso appena svolto.

Resta da chiedersi se sia più appropriata la non presunzione di colpevolezza espressa dalla nostra Costituzione o la presunzione di innocenza affermata dalla Convenzione europea. A nostro avviso, muovendo dal presupposto che entrambe le formule esprimano regole legali né ovvie né irragionevoli, occorre distinguere, a seconda che sia stata emanata o no una condanna. Prima della pronuncia di una condanna è più corretto parlare di 'presunzione di innocenza'; o meglio, sarebbe del tutto pleonastico prevedere una 'non presunzione di colpevolezza'. Come si potrebbe, infatti, ritenere colpevole colui che nessun giudice ha ancora dichiarato tale? A negare l'equivalenza è una regola logica, così elementare da non avere alcuna necessità di essere tradotta in regola legale.

Si spiega così che la Convenzione europea, riferendosi solo al periodo antecedente alla condanna, abbia imposto il più pregnante, e non ovvio, principio della presunzione di innocenza; la quale assume il valore di una regola legale, proprio perché dal punto di vista puramente logico non sarebbe corretto presumere l'innocenza per il solo fatto che non vi sia ancora la prova della colpevolezza (costituirebbe un buon esempio della c.d. fallacia ad ignorantiam).

Diverso il discorso dopo la pronuncia della condanna, seppure non irrevocabile. Qui stabilire con una regola legale che l'imputato non è considerato colpevole, lungi dall'essere pleonastico, ha il senso ben preciso di vietare un'assimilazione che, altrimenti, scaturirebbe automaticamente dalla decisione che dichiara colpevole l'imputato. In questi termini si 
esprime l'art. 27 comma 2 Cost., che opera anche nelle fasi processuali successive alla condanna non irrevocabile, dove sarebbe eccessivo pretendere di capovolgere in presunzione di innocenza l'affermazione di colpevolezza contenuta nella sentenza.

In altre parole, per attribuire alle due formule un senso non banale, si deve ritenere che entrambe istituiscano una ragionevole finzione. Prima della condanna la regola legale, ossia la finzione, sta nel presumere l'innocenza, quando in senso logico vi sarebbe spazio solo per la non presunzione di colpevolezza. Dopo la condanna la regola legale e quindi la finzione sta nel non presumere quella colpevolezza che in via logica sarebbe corretto affermare perché espressa in una valida sentenza. Per la prima ipotesi è appropriata la formula della Convenzione, per la seconda quella della Costituzione. È probabilmente per il suo riferimento anche alla fase delle impugnazioni contro la condanna che la nostra Costituzione parla di un imputato 'non considerato colpevole', anziché 'considerato innocente' (o 'non colpevole').

\section{AMMISSIBILITÀ ED EFFICACIA PERSUASIVA DELLA PROVA}

Le questioni relative all'ammissibilità della prova vanno tenute ben distinte da quelle relative all'efficacia persuasiva che in concreto può esercitare il singolo dato. Purtroppo nei discorsi sulla prova si registra spesso una confusione tra i due piani. Analizziamo, ad esempio, l'enunciato ' $x$ non è una prova'. Correttamente inteso, significa che $x$ non costituisce una valida premessa probatoria, e quindi non è valutabile dal giudice sul tema $y$ (ad esempio, perché raccolto senza l'osservanza delle regole del contraddittorio o illegittimamente acquisito al processo). Ma, nel senso improprio in cui talora è usato specie nel linguaggio corrente, sta a significare che $x$, pur costituendo una valida premessa, è inidoneo nel caso concreto a 'provare' $y$ (ad esempio, una testimonianza ritenuta inattendibile o irrilevante). C'è una differenza abissale tra dire che ' $x$ è (o non è) prova sul tema $y$ ' e dire che ' $x$ prova o non prova $y^{\prime}$ : nel primo senso si afferma o si nega una potenzialità probatoria; nel secondo, si afferma o si nega l'esito positivo della prova. La prova su y (premessa probatoria) non va confusa con la prova di y (risultato probatorio). 
Dovrebbe essere chiaro, infatti, che una testimonianza non cessa di essere 'prova' per il solo fatto che sia vaga, irrilevante o persino falsa; così come costituisce una prova la circostanza che l'imputato fosse in buoni o cattivi rapporti con la vittima, ne fosse o no geloso, pur trattandosi di un elemento di per sé assolutamente inidoneo a provare il gesto omicida che gli è imputato. 'Prova' è tutto ciò che sia legittimamente acquisito al processo e, quindi, valutabile dal giudice in ordine ad un qualsiasi tema probatorio; e poco importa, ai fini del concetto di prova, che un dato valutabile si riveli in concreto più o meno persuasivo, più $o$ meno rilevante rispetto alla proposizione da provare.

È netta la differenza tra una prova vietata dalla legge, quindi non acquisibile al processo, e una prova manifestamente irrilevante o sovrabbondante. A norma dell'art. 190 c.p.p. il giudice esclude «le prove vietate dalla legge e quelle che manifestamente sono superflue o irrilevanti». Il tenore della disposizione può indurre a ritenere che tanto le prove vietate dalla legge quanto quelle manifestamente irrilevanti o superflue siano inammissibili, dovendo il giudice non ammetterle.

Ma non è così. Inammissibile nel significato proprio della parola è la prova vietata dalla legge, ossia oggetto di una regola di esclusione probatoria (ad esempio, gli scritti anonimi, la testimonianza dell'imputato, i mezzi che ledono la libertà di autodeterminazione, ecc.); vale a dire, la prova che il giudice non ha il potere di assumere. Quella che il giudice non ammette perché manifestamente irrilevante o superflua non è inammissibile nel senso appena specificato; né sarebbe corretto dire che il giudice sia privo del potere di assumerla. Viene (provvisoriamente) esclusa perché ritenuta, al momento, inutile a fini decisori.

Il giudizio sulla superfluità e irrilevanza, infatti, non riguarda la prova in sé, intesa come premessa probatoria, ma il suo rapporto con il contesto in cui si colloca, e, più specificamente, con la proposizione da provare (nel caso della irrilevanza) o con le altre prove (nel caso della superfluità). È un giudizio che in qualche misura attiene al merito e la cui anticipazione in sede preliminare, ossia di ammissione della prova, si giustifica per esigenze di ragionevole durata del processo; sarebbe una perdita di tempo assumere prove superflue o irrilevanti. Ma, proprio per il rischio di sconfinamento nel merito e di perdita di imparzialità del giudice, la legge circoscrive l'estensione delle due tipologie con l'avverbio 
'manifestamente'. Si spiega così che il provvedimento di esclusione della prova manifestamente superflua o irrilevante sia 'allo stato degli atti', ossia sempre rivedibile al variare del quadro probatorio; la prova già esclusa può, al mutare del rapporto con le altre prove o in una diversa prospettiva di ricostruzione del fatto, mostrarsi successivamente utile e rilevante, mentre una prova vietata dalla legge resta inammissibile, quale che sia il contesto in cui si inserisce.

\section{EQUIVOCI IN TEMA DI INDIZI}

Ovunque vi sia una proposizione da provare, necessariamente devono esservi prove, non essendo possibile provare se non attraverso prove. L'ipotesi che qualcosa possa essere ‘provato' da entità diverse dalle prove - comunque le si chiami, elementi, indizi, ecc. - è una contraddizione in termini; se $x$ è provato da $y, y$ non può che essere una prova. Per converso, è altrettanto evidente che non tutte le "prove" sortiscono necessariamente l'effetto di "provare": vi sono prove, ossia dati valutabili dal giudice, che in concreto, a causa della loro irrilevanza o intrinseca debolezza, risultano ininfluenti sulla proposizione da provare o addirittura ne costituiscono la prova negativa.

Alla stregua di questa ovvia conclusione si può facilmente sciogliere il ricorrente interrogativo che riguarda l'art. 192 comma 2 c.p.p., secondo cui «l'esistenza di un fatto non può essere desunta da indizi a meno che questi siano gravi, precisi e concordanti». Gli 'indizi' costituiscono o no prove? Cade in una clamorosa contraddizione la relazione al progetto preliminare del codice di procedura penale quando afferma che con il richiamo «agli indizi gravi, precisi e concordanti si è voluto porre una regola che serva da freno nei confronti di usi arbitrari e indiscriminati di elementi ai quali, sul piano logico, non è riconosciuta la stessa efficacia persuasiva delle prove» ${ }^{10}$.

Se davvero gli indizi non costituissero 'prove', sarebbero oggetto di una regola di esclusione che impedirebbe ogni loro valutazione; e, anche se mille, gravi, precisi e concordanti, sarebbero a priori ininfluenti.

10 Relazione al progetto preliminare del 1978, 167 s. (l'articolo di riferimento del progetto era il 183). 
L'art. 192 c.p.p. fissa, invece, un criterio di valutazione relativo al valore, al significato che gli indizi, rectius le 'prove indiziarie' possono assumere in ordine alla proposizione da provare, subordinando il buon esito della prova ai requisiti della gravità, precisione e concordanza; ma ogni criterio di valutazione, al contrario delle regole di esclusione, presuppone la natura di prova del dato a cui si riferisce; altrimenti, quel dato non sarebbe in alcun modo valutabile, perché estraneo al quadro probatorio, giuridicamente inesistente a tale fine.

In conclusione ogni 'indizio' ex art. 192 c.p.p. costituisce prova - ossia premessa probatoria, legittimamente valutabile dal giudice - ma, isolatamente considerato, non può produrre l'effetto di provare. Negare natura di prova agli indizi equivale a confondere la prova, come dato valutabile, con la questione del valore che in concreto può assumere ${ }^{11}$. Le prove restano tali anche quando abbiano esito negativo; la qualifica di prova è logicamente antecedente alla sua valutazione e all'effetto che esercita sulla proposizione da provare.

In analogo errore cadono la Corte costituzionale e la Cassazione, quando affermano che nei provvedimenti cautelari il giudizio prognostico ex art. 273 c.p.p. è condotto «non su prove, ma su indizi» ${ }^{12}$. Se ai fini delle misure cautelari non occorressero prove, bisognerebbe concludere che sul tema cautelare non vi sia nulla da provare, posto che, come si accennava, solo le prove possono 'provare'. Conclusione irragionevole, essendo evidente che ogni provvedimento cautelare esige la doppia prova dei pericula libertatis (pericolo di inquinamento delle prove, di fuga o della commissione di gravi delitti) e del fumus boni iuris. Si obietterà a

11 Incappa in questa confusione Cass. 10 luglio 1996, in Guida al diritto, 2002, fasc. 48, 84: «La prova indiziaria è costituita dal complesso dei singoli indizi, ciascuno dei quali, quindi, non può avere da sé carattere esaustivo, giacché, altrimenti, sarebbe una prova e non un indizio. Non ha senso perciò affannarsi a dimostrare, per ogni indizio, che lo stesso non può essere assunto a base di un giudizio di responsabilità, in quanto suscettibile di interpretazioni diverse, perché questa è appunto la caratteristica che distingue l'indizio dalla prova e che sussiste, quindi, necessariamente, anche quando l'indizio, come previsto dall'art. 192 comma 2, sia 'grave' e 'preciso' (oltre che 'concordante' con gli altri indizi)».

12 V. Corte cost. sentenza n. 131 del 1996; nello stesso senso, per la contrapposizione tra indizi e prove, Corte cost. sentenza n. 432 del 1995, nonché Cass., Sez. un., 30 ottobre 2002, Vottari, in Cass. pen., 2003, 396, nt. 112. 
quest'ultimo riguardo che la legge parla di «gravi indizi di colpevolezza» e non di 'prove'. Ma, in base alla struttura triadica della prova, è facile rispondere che la formula "gravi indizi di colpevolezza» di cui all'art. 273 c.p.p., traducibile in 'probabile colpevolezza' o espressioni affini, non indica le premesse probatorie, ma la proposizione da provare, per la cui verifica naturalmente servono prove ${ }^{13}$.

In altri termini, tanto per la condanna quanto per i provvedimenti cautelari occorrono prove: tuttavia, mentre per la prima la proposizione da provare è la 'colpevolezza', per i secondi è solo la 'probabile colpevolezza'. E il medesimo discorso vale per i «gravi indizi di reato» richiesti per le intercettazioni di conversazioni (art. 267 c.p.p.) ${ }^{14}$ : anch'essi, esattamente come i gravi indizi di colpevolezza, non costituiscono le premesse probatorie, ma la proposizione da provare.

\section{Premesse Probatorie: PRove DiRetTe e PRove indirette}

Le prove, intese come premesse probatorie, sono oggetto di molteplici classificazioni, tre delle quali assumono particolare rilievo: prove dirette/indirette; prove costituite/precostituite; dichiarazioni di prova/prove critico-indiziarie.

Nel linguaggio corrente si parla a volte di "prova diretta" con riguardo ai fatti che cadono sotto la percezione di chi li osserva: ad esempio, la prova diretta che "fuori piove" si avrebbe uscendo per accertarlo: così come la prova delle reali dimensioni di un oggetto si otterrebbe ispezionandolo. La prova diretta sarebbe insomma la "prova" del dottor Johnson, il lessicografo che, a dimostrare la realtà di una pietra, la prese a calci, confutando così la teoria solipsistica di Berkeley dell'inesistenza del mondo materiale.

Se si dovesse accettare questa nozione di prova diretta, bisognerebbe concludere che nel processo penale tutte le prove sono indirette perché il contenuto delle proposizioni da provare non cade mai sotto la

\footnotetext{
13 Infra, par. 20.

14 In proposito v. A. CAmon, Le intercettazioni nel processo penale, Giuffrè, Milano, 1996, $68 \mathrm{~s}$.
} 
diretta percezione del giudice; e meno che mai il fatto di reato di cui il giudice non può essere testimone. Di prova diretta si potrebbe parlare solo in rapporto all'ispezione svolta dal giudice stesso per verificare la realtà di un determinato oggetto. Qui, tuttavia, è preferibile escludere dalla nozione di prova il passaggio dalle percezioni all'oggetto percepito. Anzitutto perché il rapporto tra le percezioni - intese come stimolazioni sensoriali o irritazioni di superficie determinate dal contatto col mondo esterno - e le credenze che ne derivano non è di tipo epistemico o probatorio, ma causale. Le sensazioni possono essere le cause, ma non le ragioni delle proposizioni in cui si esprimono le nostre conoscenze, giacché la prova ha sempre carattere proposizionale; e, come dice Davidson, nulla può valere come giustificazione di una credenza se non un'altra credenza ${ }^{15}$.

In secondo luogo, perché sarebbe fonte di fastidiose complicazioni terminologiche affermare che l'ispezione di un oggetto è "prova" dell'oggetto stesso o che l'ascolto orale di un teste nel dibattimento è "prova" della testimonianza. Si dirà più semplicemente che il giudice traduce in enunciati osservativi i fatti e le cose direttamente percepite, assumendoli come premesse probatorie.

Di conseguenza, se nel processo si vuol parlare di prove dirette (o di primo grado) e di prove indirette (o di secondo, terzo, quarto grado, ecc.), la distinzione va reimpostata in questi termini. Per prove dirette si intendono quelle in cui tra la proposizione probatoria e la proposizione da provare non si interpongono proposizioni intermedie: ad esempio, il teste afferma $x$ e $x$ è la proposizione da provare. Prove indirette si intendono quelle in cui tra la proposizione probatoria e la proposizione da provare si innestano proposizioni intermedie che, messe in sequenza, assumono una doppia veste: proposizioni da provare rispetto alle proposizioni precedenti; premesse probatorie rispetto a quelle seguenti. Ad esempio, la testimonianza di $N$ che afferma di avere appreso $x$ da $P$ è prova diretta quanto al fatto della narrazione ricevuta da $P$, ma indiretta rispetto al fatto $x$. E così un'intercettazione tra $N$ e $P$ che raccontano $y$ è prova diretta quanto al dialogo tra $N$ e $P$, ma indiretta rispetto ad $y$.

15 D. Davidson, Una teoria coerentista della verità e della conoscenza (1983), in A. Bottani-C. Penco (a cura di), Significato e teorie del linguaggio, Bompiani, Milano, 1991, 116. 
In realtà, più che una singola prova, la prova indiretta costituisce una combinazione di due o più prove che si dispongono in sequenza $\mathrm{o}$, più precisamente, ad incastro ${ }^{16}$ : la proposizione provata dalla c.d. prova indiretta si converte in premessa probatoria rispetto ad una nuova e diversa proposizione da provare. 'Testimonianza indiretta' è un'espressione riassuntiva con la quale si designano due racconti, quello di primo grado che narra un avvenimento e quello di secondo grado, collocato a un livello superiore, che è un metaracconto, ossia un racconto sul racconto. Da notare che, dal punto di vista strettamente linguistico, la prospettiva potrebbe essere invertita. Il metalinguaggio è un linguaggio in cui si parla di un altro linguaggio, con la conseguenza che il metaracconto dovrebbe essere il racconto primo, al cui interno se ne colloca un secondo, il racconto-oggetto. Ma in questa sede è preferibile seguire l'opinione corrente che considera di secondo grado il racconto sul racconto.

\section{Prove precostituite e prove costituite}

Prove costituite sono quelle formate all'interno della sede processuale latamente intesa, che include anche l'attività svolta dagli organi inquirenti: dichiarazioni rese da testi ed imputati, ricognizioni, confronti, perizie, esperimenti giudiziali, ecc. Prove precostituite sono tutte le altre, ossia le prove appartenenti ad una realtà esterna al processo (cose pertinenti al reato, dichiarazioni extraprocessuali, documenti, fotografie, videoregistrazioni, impronte digitali, e, più in generale, qualsiasi accadimento del mondo esterno che possa assumere rilevanza per la prova del fatto imputato). Quando si tratti di cose materiali, sono immesse nel processo - "acquisite" in termini tecnici - mediante un'attività di apprensione come il sequestro, talora preceduto dalla perquisizione; $\mathrm{o}$, quando si tratti di discorsi, tramite mezzi come la testimonianza indiretta di chi li abbia percepiti, la registrazione su supporto magnetico da parte di uno degli interlocutori o l'intercettazione ${ }^{17}$.

16 Infra, par. 13.

17 La dicotomia, qui menzionata, tra prove precostituite e prove costituite corrisponde, nel linguaggio processualcivilista, a quella tra prove costituite e prove costituende. 
Prove precostituite e prove costituite si intrecciano spesso tra loro. È il caso della testimonianza indiretta che, nella sua forma tipica, è il racconto di un racconto (per questo si chiama anche testimonianza de relato o di secondo grado o, ancora, meta-testimonianza). La testimonianza indiretta è prova costituita quanto al racconto del teste indiretto che depone nel processo, ma è prova precostituita quanto al racconto riferito; naturalmente, il teste diretto può essere chiamato a deporre nel processo, su istanza della parte o d'ufficio dal giudice, nel qual caso si avrà un terzo racconto che, al pari di quello del teste indiretto, è prova $\operatorname{costituita}^{18}$. Analoga sovrapposizione si ha nell'intercettazione, che è prova costituita quanto al supporto su cui è registrata la conversazione, ma prova precostituita quanto alla conversazione stessa, che si è svolta fuori dal procedimento ${ }^{19}$.

\section{DICHIARAZIONI DI PROVA E PROVE CRITICO-INDIZIARIE}

Molto importante è la distinzione tra dichiarazioni di prova (o prove storiche o funzioni narrative) e prove critico-indiziarie, in ordine alla quale va subito premesso che il secondo termine non coincide, anzi ha poco a che spartire con il concetto di 'indizi' che traspare dall'art. 192 comma 2 c.p.p. .

Per comprendere il senso dell'antitesi si prenda una qualsiasi proposizione da provare, ad esempio quella che descrive il fatto $x$, oggetto di prova. La prova del fatto $x$ può svolgersi in due diversi modi o tecniche di prova ${ }^{20}$ :

a) attraverso la narrazione di qualcuno che lo asserisce come vero e se ne rende per così dire garante (è la figura tipica del testimone, ma, più

18 Per le ragioni già esposte è preferibile considerare ogni racconto come prova; e, quindi, la testimonianza indiretta come sequenza di due prove dichiarative (v. anche infra, par. 14).

19 Sulla complessa struttura dell'intercettazione, v. infra, par. 12.

20 Sulla distinzione v. soprattutto F. CARnElutTi, La prova civile [1915], Giuffrè, Milano, 1955, 92 s.; F. Cordero, Procedura penale, IX ed., Giuffrè, Milano, 2012, 576 s.; ma cfr. anche F. CAPRIOLI, Colloqui riservati e prova penale, Giappichelli, Torino, 2000, 298 s.; P. FERrUA, Studi sul processo penale, vol. II, Giappichelli, Torino, 1992, 53 s. 
in generale, di chiunque affermi qualcosa a titolo di verità nell'ambito di un discorso che non sia di finzione); quindi attraverso un processo comunicativo fondato sulla trasmissione di un sapere attraverso i segni linguistici;

b) attraverso un qualsiasi altro fatto $y$, diverso dalla narrazione di $x$, dal quale si possa, mediante un ragionamento critico, fondato sulla scienza o sull'esperienza corrente, indurre il fatto $x$.

Chiariamo il concetto con un esempio, del tutto indipendente dal processo penale, ma significativo. Che ieri piovesse nel tal luogo si può 'provare' attraverso qualcuno che lo afferma o mediante le tracce di bagnato rimaste sul terreno. Nel primo caso la prova è dichiarativa (detta anche "dichiarazione di prova" o "funzione narrativa"), in quanto il fatto da provare è già espresso con i segni linguistici dalla stessa premessa probatoria (dove si afferma che ieri pioveva); nel secondo è critico-indiziaria, perché il fatto da provare non è verbalizzato nella premessa probatoria ma da questa indotto - rectius, abdotto - in base a relazioni di causa-effetto (l'acqua sul terreno è il segno naturalistico e non linguistico della pioggia).

La differenza strutturale è chiara. La prova dichiarativa contiene già in sé la descrizione del fatto da provare, per cui l'esito positivo o negativo dipende dalla fede che si presta al dichiarante. In questo genere di prova la proposizione da provare si limita ad oggettivare e a sanzionare come vero ciò che nella premessa probatoria è asserito da qualcuno (nell'ipotesi tipica, il teste). Se la premessa probatoria è "Tizio afferma che $x$ ", la proposizione da provare è " $x$ " (o, se si preferisce, "è vero che $x$ "); la seconda proposizione è già interamente contenuta nella prima. Nella prova critico-indiziaria, invece, la proposizione da provare è inespressa e va individuata con un'attività di decifrazione, più o meno complessa, che implica competenze di tipo empirico, tratte dalla scienza e dall'esperienza corrente. Tutte le prove che non sono dichiarative sono per esclusione critico-indiziarie; non esistono categorie intermedie.

\section{CRiteri identificativi della prova dichiarativa. L'atto COMUNICATIVO}

Correttamente intesa, la prova dichiarativa poggia, dunque, su due connotati. Il primo è di essere un atto comunicativo, volto a trasmettere intenzionalmente un'informazione attraverso segni linguistici, ossia con 
mezzi idonei a significare nel senso non naturalistico dell'espressione: significato naturale è quello che compare in frasi come le nuvole significano pioggia, o la febbre significa influenza, mentre significato non naturale è quello che compare in frasi come pluie significa pioggia o il suono del campanello significa la fine della lezione o i rintocchi della campana significano le ore ${ }^{21}$.

La prova dichiarativa, specie quella costituita nel processo, si svolge di regola attraverso il linguaggio verbale che per definizione è un mezzo comunicativo. Ma, fuori dal processo, un'informazione può essere intenzionalmente trasmessa anche attraverso segni non verbali, idonei a funzionare come un linguaggio (gesti, colori, fiori, bandiere, disegni, ecc.); nel qual caso non sempre è facile comprendere se si sia o no in presenza di un intento comunicativo e, quindi, rispettivamente, di una prova dichiarativa o di una prova critico-indiziaria. Stabilire se un comportamento non verbale sia o no 'comunicativo' assume una precisa rilevanza agli effetti delle videoregistrazioni in ambienti domiciliari: come si sa, la Corte costituzionale ha ritenuto che l'autorità giudiziaria possa disporle, applicando la disciplina delle intercettazioni ambientali, solo se destinate a riprodurre contegni di tipo comunicativo ${ }^{22}$.

Il criterio di demarcazione, chiaro a livello teorico ma di non semplice applicazione pratica, è quello indicato da Paul Grice, così riassumibile: $S$ ha voluto dire qualcosa con $x$ quando $S$ ha avuto l'intenzione che $x$ producesse un certo effetto sull'ipotetico destinatario attraverso il riconoscimento di questa intenzione ${ }^{23}$. Solo a queste condizioni si può

21 Mentre il significato non naturale può essere indicato tra virgolette, l'indicazione sarebbe priva di senso per quello naturale: possiamo dire che pluie significa "pioggia" o che il verde del semaforo significa 'via libera', ma non diremmo che le nuvole significano "pioggia" o che la febbre significa 'influenza'.

22 Corte cost. sentenza n. 135 del 2002. Sul tema v. Bozio, La prova atipica, in P. Ferrua-E. Marzaduri-G. Spangher (a cura di), La prova penale, Giappichelli, Torino, 2013, $77 \mathrm{~s}$.

23 P. Grice, Logica e conversazione (1989), trad. it., Il Mulino, Bologna, 1993, 58.

Così, sfogliando un album di fotografie posso trasmettere a chi mi è accanto informazioni, senza intento comunicativo; ma, se eseguissi dei disegni sui medesimi oggetti, realizzerei un contegno comunicativo. La fotografia, infatti, non è, almeno di regola, un mezzo comunicativo, il disegno può esserlo. Nel disegno, infatti, la cosa rappresentata, sussista o no nel reale, deriva interamente 
dire che con $x$ si sia realizzato un atto comunicativo. E naturalmente se, a fini probatori, $x$ (in ipotesi, un gesto o altro contegno non verbalizzato) funzioni in senso dichiarativo o critico-indiziario dipende da come lo decifra il giudice, a seconda che vi legga o no l'intento comunicativo.

Preliminare alla valutazione della prova dichiarativa è, dunque, l'esatta individuazione dell'informazione che l'emittente intende trasmettere attraverso il riconoscimento della sua intenzione di trasmetterla. Non costituiscono prove dichiarative le emissioni verbali in stato di incoscienza (sonnambulismo, narcoanalisi); e, in base ad un'elementare regola di civiltà codificata nell'art. 188 c.p.p., altrettanto dicasi per quelle rese in stato di costrizione fisica o psichica.

Perché si realizzi un atto comunicativo occorrono, dunque, tre condizioni: $a$ ) la trasmissione di un'informazione; $b$ ) l'intenzione di trasmettere l'informazione; $c$ ) l'intenzione che l'informazione sia ricevuta attraverso il riconoscimento dell'intenzione di trasmetterla. Essendo sufficiente la doppia intenzionalità dell'emittente (in rapporto a $b$ e $c$ ), resta, invece, irrilevante che la comunicazione giunga a buon esito, ossia che il destinatario riceva il messaggio e riconosca l'intento comunicativo: anche ciò che si scrive in un appunto o in diario è atto comunicativo, indipendentemente dalla circostanza che qualcuno lo legga.

Esemplifichiamo i concetti, immaginando che Tizio, seduto al tavolo di un ristorante, agiti il bicchiere vuoto. Possiamo distinguere le seguenti situazioni. Nella prima il soggetto, utilizzando il gesto come un linguaggio, intende apertamente trasmettere al personale l'invito a servirgli da bere; in tal caso si realizzano le tre componenti della comunicazione, anche se, come vedremo, questa non è di tipo propriamente dichiarativo, trattandosi di un ordine che in sé non è né vero né falso.

dall'intenzionalità di chi la disegna; nella fotografia (s'intende, non truccata) la cosa rappresentata, necessariamente reale, 'è stata' davanti all'obiettivo e i suoi raggi luminosi si sono impressi sul supporto che, attraverso meccanismi chimici o elettronici, ha prodotto l'immagine. Come scriveva Roland Barthes, a differenza del disegno o della pittura che possono «simulare la realtà senza averla vista», la fotografia «è letteralmente un'emanazione del referente. Da un corpo reale, che era là, sono partiti dei raggi che raggiungono me, che sono qui» (La camera chiara [1980], trad. it., Einaudi, Torino, 2003, 77). Naturalmente, il 'realismo' della fotografia non deve indurre a confondere l'immagine con il referente di cui costituisce solo un effetto, una traccia: v infra, par. 12. 
Nella seconda Tizio, senza palesare le sue intenzioni, vuole indurre il personale a servirgli da bere, col proposito che il suo gesto non sia recepito come una richiesta, ma sia decifrato come un segno naturale (quindi, critico-indiziario) di sete: qui si realizzano le prime due condizioni (la trasmissione di un'informazione e l'intento informativo), ma manca l'ultima condizione dell'atto comunicativo, perché l'informazione è trasmessa senza la volontà che sia riconosciuto l'intento informativo.

Nella terza, infine, il soggetto agita il bicchiere perché assetato o, semplicemente, perché nervoso, ma senza alcuna intenzione informativa: in tal caso, si realizza solo la prima condizione, perché il gesto, pur idoneo a tramettere varie informazioni in chiave critico-indiziaria, è slegato da qualsiasi proposito informativo. È evidente che solo nel primo caso il gesto funziona propriamente come un linguaggio, ossia trasmette significati nel senso non naturalistico della parola.

Trattandosi di un criterio imperniato sulle intenzioni dell'emittente, non è sempre agevole accertare se il gesto abbia o no carattere comunicativo: il solo mezzo per decifrarlo è un'attenta analisi del contesto.

\section{L'ENUNCiATO APOFANTICO}

Perché la prova sia dichiarativa non è sufficiente che si risolva in un atto comunicativo. Occorre che gli enunciati dell'atto comunicativo siano veri o falsi. L'essenza della prova dichiarativa sta nel certificare come vero ciò che si asserisce, indipendentemente dall'essere o no previste sanzioni per il falso. La proposizione da provare - che per definizione è vera o falsa - dev'essere già interamente espressa nella premessa probatoria, con la conseguenza che costituiscono prove dichiarative solo gli enunciati apofantici, ossia soggetti all'alternativa vero/falso. Non sono tali i comandi, i suggerimenti, i consigli, le minacce, le promesse e, in generale, tutto il discorso di finzione o di fantasia (romanzi, favole, fantascienza, ecc.), per il fatto stesso che, non essendo né veri né falsi, non possono asserire la proposizione da provare. Ogni atto dichiarativo è, al tempo stesso, comunicativo, ma non ogni contegno comunicativo veicola dichiarazioni.

Questo non significa che gli enunciati non soggetti all'alternativa vero/falso siano privi di valore probatorio; ma più semplicemente che 
saranno valutabili in chiave critico-indiziaria rispetto ad una proposizione diversa da quella (né vera né falsa) in essi verbalizzata. Ad esempio, una minaccia o un ordine, pur non avendo natura dichiarativa, possono assumere per il loro contenuto una notevole rilevanza probatoria nella ricostruzione dei fatti per cui si procede. La distinzione tra atti dichiarativi e non dichiarativi è essenziale per delimitare l'ambito operativo del criterio di valutazione che vieta di provare la colpevolezza sulla base delle dichiarazioni rese da chi si è sempre sottratto per libera scelta al contraddittorio (artt. 111 comma 4 Cost., 526 comma 1-bis c.p.p.).

\section{Prove CRITICo-INDIZIARIE}

Più che sulla base di specifici connotati la prova critico-indiziaria va definita in via residuale, sottraendo dall'intero sistema delle prove quella dichiarativa. Tutto ciò che non è prova dichiarativa, ossia non trasmette informazioni attraverso il linguaggio (sia o no verbale), è per esclusione prova critico-indiziaria: tali sono le fotografie, le videoregistrazioni, le impronte digitali, il dna, gli oggetti pertinenti al reato, e, più in generale, qualsiasi fatto che abbia rilevanza induttiva ai fini della prova del reato.

La prova critico-indiziaria è in senso propriamente comunicativo muta, perché non esprime né descrive il fatto da provare, ma ne è semmai il segno naturale, la traccia non linguistica. La prova indiziaria - come suggerisce l'etimo (index) - è un 'indice': addita, ma non dice, non 'parla' nel senso letterale del verbo. L'aggettivo "critico-indiziaria" sta ad indicare l'attività di decifrazione che svolge il giudice per risalire dal fatto probatorio a quello da provare. In altri termini, mentre nelle funzioni narrative la proposizione da provare è già contenuta ed espressa nella premessa probatoria (la dichiarazione di prova), nella prova critico-indiziaria la proposizione da provare dev'essere individuata dai soggetti interessati, il cui impegno critico si esplica su ciò che la prova può significare nel senso naturalistico della parola, che designa per lo più relazioni di causa-effetto ${ }^{24}$.

${ }^{24}$ La circostanza che nella fotografia o nella videoregistrazione il fatto da provare sia 'rappresentato' con chiarezza non equivale a dire che sia 'enunciata' o 'espressa' la proposizione da provare. 
Più complessa la classificazione degli oggetti-utensili costruiti dall'uomo allo scopo di significare, come i segnali, le spie luminose, le maniche a vento, ecc. Qui la componente comunicativa si sovrappone a quella critico-indiziaria; ad esempio, la comparsa del verde in un semaforo può essere decifrata tanto in chiave critico-indiziaria, come l'effetto di certi impulsi elettrici, quanto in chiave comunicativa, come messaggio di via libera. Per comprendere la parte critico-indiziaria serve un sapere tecnico-scientifico, per intendere quella comunicativa una competenza semantica.

Per le ragioni già esposte sono prove di tipo critico-indiziario anche gli enunciati non soggetti all'alternativa vero/falso, come comandi, promesse, ordini, ecc.: vi è l'intento comunicativo, ma il contenuto della comunicazione, non asserendo nulla come vero, può solo costituire la premessa critico-indiziaria per una diversa proposizione da provare. Se qualcuno afferma che la porta è aperta, si è in presenza di una prova dichiarativa rispetto alla circostanza che la porta sia aperta: e, infatti, nella proposizione probatoria è già linguisticamente espressa la proposizione da provare (l'apertura della porta). Ma, se qualcuno ordina di chiudere la porta, è solo in via critico-indiziaria che dall'ordine si può indurre che la porta è aperta: la proposizione probatoria non contiene quella da provare, essendo diversi i rispettivi referenti (la chiusura e l'apertura della porta).

Da notare, infine, che per la valutazione di attendibilità delle prove dichiarative, specie di quelle assunte oralmente nell'istruzione dibattimentale, assumono grande rilevanza una serie di indici riconducibili alla logica delle prove critico-indiziarie: i tratti paralinguistici o prosodici del discorso, le lacune, i lapsus in cui sia incorso il dichiarante e, più in generale, tutta la produzione segnica non intenzionale che accompagna le emissioni verbali, così preziosa per decifrare le menzogne. A screditare un discorso, può essere, talvolta, lo stesso atteggiamento di chi lo proferisce ${ }^{25}$.

In situazioni limite, può accadere che un atto comunicativo - poco importa se dichiarativo o no - sia decifrato dal destinatario in chiave

25 Tuttavia, per quanto importante, l'influenza dei tratti prosodici sul convincimento del giudice resta per lo più confinata in sede psicologica, non essendo facile renderne conto in sede di motivazione, specie quando non sia disponibile la videoregistrazione. 
critico-indiziaria attraverso una lettura 'a rovescio' o 'di traverso', nella quale le parole sono assunte non per quel che significano, ma per ciò che possono veicolare di falso o di non espresso. È quanto capita con i maldestri mentitori, le cui modalità espressive a volte sono così eloquenti da convertire un'affermazione in negazione o viceversa; e altrettanto con le persone affette da riserve mentali o che intendono trasmettere in forma criptica un'informazione diversa da quella di cui si assumono la responsabilità come emittenti.

Inutile chiedersi se siano più affidabili le prove dichiarative o le prove critico-indiziarie. Le prime possono mentire; le seconde essere alterate, e, quando siano genuine, la loro efficacia persuasiva varia a seconda del legame che sussiste tra il fatto probatorio e il fatto da provare: più forte nel caso di prove critico-indiziarie fondate su leggi scientifiche, più debole se basate sull'esperienza corrente o su inferenze di natura psicologica.

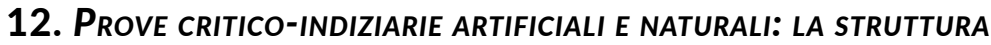 COMPLESSA DELL'INTERCETTAZIONE}

Le prove critico-indiziarie possono essere distinte in artificiali o naturali a seconda che siano o no congegnate dall'artificio umano allo scopo di provare (rectius, anche di provare, non essendo necessario che questa sia la loro unica funzione). Tra le prime, la fotografia, la registrazione di suoni o di immagini, le quali a loro volta possono essere costituite o precostituite a seconda che siano o no disposte nella sede processuale latamente intesa (ad esempio, la registrazione di un dialogo ad opera di un privato è prova precostituita, mentre è costituita in quanto sia disposta da organi inquirenti nel corso delle loro indagini, come accade con l'intercettazione). Tra le seconde, le cose pertinenti al reato, le impronte digitali e qualsiasi fatto induttivamente rilevante per la ricostruzione della condotta criminosa.

È evidente che le prove artificiali, proprio in quanto destinate a provare, sono in genere ad alta attendibilità; e non stupisce che, quando sono orientate in senso accusatorio, la difesa sia spesso indotta a ripiegare su riti alternativi in vista di una riduzione della pena. Nella registrazione di suoni o di immagini la forza di rappresentazione è tale da evocare la presenza stessa del referente nella sua materialità (persone, dialoghi o 
fatti); il passaggio dalla prova (il mezzo di rappresentazione) alla proposizione da provare (l'oggetto della rappresentazione) è così immediato e intransigente da risolversi quasi in una coincidenza, in un cortocircuito che lascia scarso spazio a controdeduzioni.

La sensazione è di una sorta di resurrezione del passato, come se la conversazione (o la scena) si presentasse nella sua realtà, priva di qualsiasi intermediario. Ma, senza negare l'elevata affidabilità di queste tecniche, occorre essere consapevoli che è un effetto illusorio: nella migliore delle ipotesi si è soltanto di fronte alla traccia sonora o luminosa di ciò che 'è stato' il referente della prova; e, nella peggiore, davanti alla sua contraffazione. Come insegna il quadro di René Magritte ('Questa non è una pipa'), non si deve confondere l'immagine con la realtà, la rappresentazione con l'oggetto rappresentato. Il che, ovviamente, non esclude la differenza strutturale, già illustrata, tra disegno e fotografia (o videoregistrazione) nel rapporto con il referente ${ }^{26}$. Mentre nel disegno la cosa rappresentata, sussista o no nel reale, deriva interamente dall'intenzionalità di chi la riproduce, nella fotografia o nella videoregistrazione (s'intende, non truccata) il referente, necessariamente reale, 'è stato' davanti all'obiettivo che ne ha prelevato, per così dire, l'impronta.

$\grave{E}$ in questa chiave che va analizzata, nella sua complessità, l'intercettazione di conversazioni (o, mutatis mutandis, la videoregistrazione $)^{27}$. Il codice la classifica, insieme alle ispezioni, perquisizioni e sequestri, come semplice mezzo di ricerca della prova, probabilmente per effetto di quell'illusione "referenziale" che porta ad assimilare la registrazione di una conversazione al prelievo di un oggetto che si esegua nel corso della perquisizione. In realtà, mentre la perquisizione porta all'apprensione di qualcosa che preesiste ed è quindi indipendente dall'attività di ricerca, l'intercettazione non 'preleva' la conversazione, ma ne costituisce la traccia impressa sul supporto. La traccia, quando non sia alterata, è l'effetto della conversazione che si è svolta; e, poiché questa, come evento del passato, non è più disponibile, la riproduzione sonora (o visiva) ne diventa la prova; con un'inferenza di carattere

26 V. retro, la nota 23.

27 V. ampiamente, A. CAmon, Le intercettazioni, cit., 14 s.; F. CAPrioli, Colloqui riservati, cit., 2000. 
'abduttivo', tipica del processo penale, dal fatto del presente (i suoni registrati) si ricava quello del passato (la conversazione nel reale); dall'effetto, ottenuto grazie alla mediazione dell'artificio umano, si risale alla causa.

Con la perquisizione si trova un oggetto da sequestrare, con l'intercettazione si elabora, si costruisce la prova di una conversazione. Naturalmente nessuno può negare che l'inferenza, con cui dall'ascolto del nastro (o dalla visione filmica) si risale alla realtà della conversazione (o della scena registrata), sia di altissima attendibilità, tale da apparire quasi inconfutabile, salve le possibili contraffazioni; e per questa ragione, come si notava, quando la prova a carico deriva da intercettazioni o (videoregistrazioni), la difesa ripiega spesso su un rito negoziale, in vista di una riduzione della pena.

In sintesi, nell'intercettazione si possono concettualmente isolare tre profili. Quanto allo strumento che capta la conversazione, l'intercettazione è, al tempo stesso, mezzo di ricerca e di formazione della prova; quanto al supporto magnetico, su cui è registrata la conversazione, è prova critico-indiziaria, formata in sede processuale, della conversazione stessa; la quale, a sua volta, è prova dichiarativa, formata fuori dal processo, di ciò che viene in essa affermato (o prova critico-indiziaria relativamente agli enunciati né veri né falsi).

\section{3. "Sequenze" e "convergenze" probatorie}

Come accade per le prove precostituite e costituite, anche le dichiarazioni di prova e le prove critico-indiziarie possono combinarsi in vario modo. Si dispongono 'a raggera', quando convergono verso la medesima proposizione da provare; nel qual caso si irrobustiscono reciprocamente, rafforzando l'esito positivo della prova: ad esempio, un'impronta digitale e una testimonianza concordano nel segnalare il passaggio dell'imputato in un certo luogo e, insieme, assumono un valore probatorio superiore a quello della loro semplice addizione. Si dispongono 'in sequenza', quando la prima proposizione provata diventa premessa probatoria rispetto ad una nuova proposizione da provare, e così di seguito; nel qual caso, la forza probatoria scema al crescere dei passaggi, perché ognuno di essi sconta la fallibilità dei precedenti. 
Si possono ipotizzare quattro tipi di combinazione "a sequenza":

a) una prova critico-indiziaria su una dichiarazione di prova: ad esempio, la registrazione (prova critico-indiziaria) di un dialogo (prova dichiarativa) o la fotocopia di un documento scritto;

b) una dichiarazione di prova su una prova critico-indiziaria: ad esempio, la testimonianza di qualcuno che asserisca (prova dichiarativa) di avere visto l'imputato aggirarsi sul luogo del reato (prova critico-indiziaria del reato);

c) una prova critico-indiziaria su una prova critico-indiziaria: ad esempio, una fotografia o una videoregistrazione (prove critico-indiziarie del fatto rappresentato) che documentino la presenza dell'imputato in prossimità del luogo del reato (prova critico-indiziaria del reato);

d) una prova dichiarativa su una prova dichiarativa: ad esempio, la testimonianza indiretta $\mathrm{o}$, più generale, il racconto di un racconto.

\section{Proposizioni da provare}

Nell'ambito delle proposizioni da provare occorre praticare una doppia distinzione. La prima è tra le proposizioni finali, che sono quelle già individuate dal legislatore come oggetto di prova, e le proposizioni intermedie in cui può eventualmente articolarsi la sequenza probatoria, nel senso già illustrato. La seconda, che opera all'interno delle proposizioni finali, è tra la proposizione principale, che è rappresentata dalla colpevolezza, e le proposizioni incidentali costituite da altri temi di prova su cui il giudice può essere chiamato a decidere: ad esempio, la condotta illecita ex art. 500 comma 4 c.p.p., i gravi indizi di colpevolezza e i pericula libertatis nei provvedimenti cautelari (artt. 273-274 c.p.p.), i gravi indizi di reato nelle intercettazioni di conversazioni (art. 267 c.p.p.).

Il tenore delle proposizioni finali contribuisce, insieme al rito prescelto, a definire il materiale probatorio utilizzabile a fini decisori. Viene qui in rilievo il c.d. relativismo probatorio per effetto del quale ciò che è prova sul tema $x$, può non esserlo sul tema $y$ : ad esempio, per la colpevolezza vale la regola del contraddittorio nella formazione della prova (con le eccezioni previste dalla Costituzione), mentre per i temi cautelari sono utilizzabili anche prove raccolte unilateralmente. E ancora, 
dato che ogni processo va svolto e definito nei confronti di singoli imputati (anche nel caso in cui fosse disposta la riunione dei giudizi), ciò che è valutabile come prova nei riguardi di un imputato, può non esserlo nei confronti di un altro: si pensi all'incidente probatorio al quale sia rimasta estranea la difesa di un coimputato.

Mentre la proposizione principale, in quanto rappresentata dalla colpevolezza per il fatto imputato, è facilmente identificata, non è sempre agevole estrarre dal continuum della legge processuale le proposizioni relative ai temi incidentali. Accade così che, non essendo individuata la proposizione da provare, si aprano interminabili discussioni in ordine alle regole di giudizio da seguire su quei temi. Dispute improduttive, anzi fuorvianti. Non ha senso interrogarsi sulla regola da seguire se prima non si è identificato con chiarezza l'oggetto del giudizio: il 'cosa provare', ossia la ricerca della proposizione da provare, è logicamente anteriore al 'come provare', ossia alla regola di giudizio che indica a quali condizioni la prova sia raggiunta. La confusione tra i due aspetti alimenta la fallace idea che sui temi incidentali operino regole di giudizio alternative a quella che vale per il tema principale, mentre la differenza nella prova dei vari temi va ricercata nella proposizione da provare; non nella regola di giudizio che - ove non sia diversamente disposto - è una sola, imposta dalla logica.

Occorre, dunque, individuare con assoluta priorità la proposizione da provare, che è veicolata dal termine 'marcato' di ogni alternativa decisoria $^{28}$. Sta qui la maggiore difficoltà, essendo a volte problematico stabilire quale, fra i termini dell'alternativa, contenga la proposizione da provare. Ad esempio, nessun dubbio che nell'alternativa condanna proscioglimento il termine marcato sia la condanna, che contiene per l'appunto l'affermazione della colpevolezza; meno semplice, come si vedrà, individuare il termine marcato e, di conseguenza, la proposizione da provare, nell'alternativa tra il non luogo a procedere e il rinvio a giudizio o nei provvedimenti cautelari

Identificata la proposizione da provare, la regola di giudizio, che risolve i casi di incertezza, è molto semplice e di generale applicazione. Salvo espressa deroga, nessuna proposizione, che costituisca tema, principale o incidentale, del processo, può essere affermata dal giudice se non ne sia

28 Sulla distinzione tra termine marcato e termine consequenziale v. infra par. 21. 
data la prova piena, in tutte le sue componenti; e 'prova piena' significa invariabilmente prova 'oltre ogni ragionevole dubbio' 29 . È una regola logica che non esige d'essere codificata; anzi, tradurla in legge relativamente a singoli temi può riuscire dannoso, nella parte in cui lascia supporre che, altrove, valga un diverso regime. L'intervento del legislatore ha un senso solo in quanto intenda deviare in sede processuale dalla regola appena esposta, modificando lo standard probatorio (così per la prova delle cause di giustificazione) o convertendo un fatto costitutivo in fatto impeditivo (così per le esigenze cautelari, relativamente ad alcuni gravi delitti).

\section{L'atTo del pRovare E IL RAgIONAMENTO ABDUtTIVO}

Caratteristica fondamentale della prova nel processo penale è che l'inferenza non si svolge secondo la logica deduttiva, ossia con un metodo in cui la conclusione discende necessariamente dalle premesse, secondo l'implicazione del tipo 'se A allora B', dove è impossibile che B sia falsa se A è vera; ma attraverso un meccanismo di tipo abduttivo, con il quale dai fatti del presente, ossia dalle prove, si risale al fatto del passato in cui consiste in via d'ipotesi la colpevolezza.

Tutti conoscono il celebre esempio di 'abduzione' illustrato da Peirce. Sul tavolo vi è un sacchetto aperto di fagioli bianchi e lì vicino, sparsi, alcuni fagioli bianchi. L'osservatore può 'abdurne', con tutti i rischi di errore che ciò comporta, che i fagioli sparsi provengano da quel sacchetto. C'è chi dice che nell'abduzione 'si tira ad indovinare', altri che 'si va alla ricerca della migliore spiegazione'; e c'è del vero in entrambe le prospettive. Si tira a indovinare perché nulla garantisce che i fagioli provengano dal sacchetto per il solo fatto di trovarsi in prossimità e di essere bianchi; ma al tempo stesso, se non disponiamo di altri dati, quella conclusione può apparire come la migliore, la più attendibile spiegazione; il che - si ripete - non significa ancora che sia giusta e neppure che sia una buona spiegazione; potrebbe essere semplicemente la meno debole rispetto ad altre debolissime.

Ora il metodo che nel processo penale segna il passaggio dai fatti del presente, rappresentati dalle prove, al fatto del passato, costituente in

29 Infra par. 16. 
ipotesi reato, è per l'appunto una specie del genere abduttivo. Mentre è il passato (la causa) a determinare il presente (l'effetto) - le prove sono le tracce, gli effetti del reato - il giudice è costretto ad invertire i termini dell'implicazione. Anziché muovere dall'antecedente verso il conseguente (come avviene nelle leggi scientifiche), egli procede a ritroso dal conseguente verso l'antecedente, come è tipico del ragionamento abduttivo. Se la regola di implicazione afferma che 'se è A, allora segue B', il giudice la inverte ricavando A dalla presenza di B; inferenza che, valutata dal punto di vista deduttivo, costituirebbe la c.d. fallacia dell'affermazione del conseguente.

Le prove, per solide che siano, non determinano mai la colpevolezza, possono esclusivamente sotto-determinarla, Nel linguaggio della scienza, le prove sotto-determinano le teorie, nel senso che sono compatibili con una pluralità di teorie concorrenti. Altrettanto accade nel processo: quali che siano le prove a carico, in linea puramente logica, è sempre possibile ipotizzare una spiegazione diversa dalla colpevolezza dell'imputato ${ }^{30}$.

Sta qui il dramma del processo: da un lato, vi è una forte pretesa di verità perché dalla condanna discendono pesanti conseguenze per la libertà dell'individuo e suonerebbe derisoria un'affermazione di colpevolezza che non aspirasse ad essere vera; ma, dall'altro, è una pretesa mai compiutamente realizzabile, avendo come oggetto quei fantasmi che sono i fatti del passato. Quali che siano gli strumenti offerti dal progredire della scienza, il passato non sarà mai oggetto di "scoperta", ma soltanto di "ri-costruzioni”, insuscettibili di confronto con l'originale. Si potrebbe definire il giudice come una Cassandra alla rovescia: con gli occhi fissi sul presente, profetizza le sciagure del passato. Con la differenza che chi profetizza il futuro si espone a una smentita, chi ricostruisce il passato no o, comunque, molto meno.

Come conciliare, allora, l'elementare esigenza che la colpevolezza non sia solo il frutto di ipotesi con l'impossibilità di raggiungere

30 Cfr. Ferrua, Il 'giusto processo', cit., 69 s.; Tonini-Conti, Il diritto delle prove penali, II ed., Giuffrè, Milano, 2014, 165 s. Sul carattere probabilistico delle inferenze nel processo v. F. CAPRIOLI, Verità e giustificazione nel processo penale, in Riv. it. dir. proc. pen., 2013, 623 s. 
un'assoluta certezza? Il ragionamento abduttivo di per sé non offre sufficienti garanzie, consente solo la formulazione delle ipotesi, e non basta che una di queste appaia più attendibile. Che la colpevolezza sia la migliore tra le possibili spiegazioni del materiale probatorio può essere una ragione per rinviare a giudizio, per formulare un'accusa, non certo per condannare.

Emerge qui una netta differenza tra indagine preliminare e dibattimento. L'una e l'altro reggono su un meccanismo di tipo abduttivo. $\mathrm{Ma}$, mentre nell'indagine preliminare si tratta di individuare l'ipotesi abduttiva, di mettere a punto la proposizione da provare, relativa al fatto del passato, nel dibattimento l'abduzione è ormai formulata, la proposizione da provare è già espressa. Occorre convalidarla. La colpevolezza, ipotizzata in via abduttiva, dev'essere provata; e 'provare', fuori dall'ambito deduttivo, significa verificare, giustificare la conclusione 'oltre ogni ragionevole dubbio'. Questa regola, già implicita nel concetto stesso di 'provare', è stata ufficialmente consacrata all'interno del processo penale italiano dall'art. 533 comma 1 c.p.p., come modificato dalla legge 20 febbraio 2006 n.46, meglio nota come 'legge Pecorella'.

\section{LA REGOLA DELL'OLTRE OGNI RAGIONEVOLE DUBBIO: DUE MODELLI DI PROVA}

L'essenza della regola - rectius, come vedremo, del principio sta nel sintagma "ragionevole dubbio"31. È una formula che, da un certo punto di vista, appare chiara e trasparente, d'immediata comprensione.

31 Sul senso della formula «colpevole al di là di ogni ragionevole dubbio» e sull'opportunità della sua introduzione nel sistema v. di chi scrive, Il giudizio penale: fatto e valore giuridico, in P. FERrua-F. Grifantini-G. Illuminati-R. Orlandi, La prova nel dibattimento penale, I ed., Giappichelli, Torino, 1999, 217. Per i riflessi della regola nella prova del nesso di causalità, F. STELLA, Giustizia e modernità, Giuffrè, Milano, 2001, 53 s.; G. CANzIo, L'«oltre il ragionevole dubbio» come regola probatoria e di giudizio nel processo penale, in Riv. it. dir. proc. pen., 2004, 303 s.; F. CAPRIOLI, Scientific evidence e logiche del probabile nel processo per il "delitto di Cogne", in Cass. pen., 2009, 1867 s.; ID., L'accertamento della responsabilità penale 'oltre ogni ragionevole dubbio', in Riv. it. dir. proc. pen., 2009, 51 s. Da ultimo v. ampiamente E.M. Catalano, Ragionevole dubbio e logica della decisione, Giuffrè, Milano, 2016. 
Ma da un altro - forse, proprio perché si tratta di un concetto elementare, non ulteriormente riducibile - sfida ogni definizione.

Analizziamo le due principali componenti del concetto, iniziando dal sostantivo "dubbio". Che nel processo il dubbio sulla responsabilità dell'imputato debba risolversi nella sentenza assolutoria, deriva dal fatto stesso che tema del processo non è l'innocenza ma la colpevolezza, come delineata nella fattispecie incriminatrice; ed è questa, dunque, a dover essere provata sino all'ultima molecola. Ritenere 'provata' una proposizione della quale vi è ragione di dubitare appare, già a prima vista, una palese contraddizione e, sul piano linguistico, una falsificazione semantica.

In realtà, il discorso è più complesso perché esistono due distinti modelli di prova a cui corrispondono due sotto-significati di 'provare': il modello delle scienze formali e quello delle scienze empiriche, al quale appartiene anche la prova nel processo penale. Il primo è il modello della prova intesa come 'dimostrazione', dove le premesse 'determinano', ossia implicano deduttivamente la conclusione, non essendo possibile che le prime siano vere e falsa la seconda; qui la prova ben può dirsi raggiunta 'oltre ogni dubbio', con una forza tale da imporsi come necessaria per ogni persona cognitivamente lucida.

Il secondo è il modello della prova intesa come argomentazione, in cui le premesse 'sotto-determinano' la proposizione da provare, ossia la rendono più o meno probabile, più o meno fondata, non essendovi garanzia che l'essere provato come vero corrisponda necessariamente all'essere vero. Il passaggio dalle premesse alla proposizione da provare non avviene sulla base di regole linguistiche e connessioni logiche, come nella dimostrazione, ma in base a rapporti causali.

A quest'ultimo modello appartiene la prova nel processo penale. Quale che sia il grado di evidenza presente nel singolo processo, è sempre possibile mettere in dubbio, contestare la colpevolezza affermata dal giudice di merito. Se la colpevolezza dovesse discendere deduttivamente dalle prove, così da riuscire inconfutabile per qualsiasi persona razionale, ogni processo finirebbe con l'assoluzione. L'astuto penalista uscirebbe sempre vittorioso nel sostenere che manca la prova indubitabile della commissione del fatto, del dolo dell'imputato, della sua capacità di intendere e volere, ecc.

Di qui l'esigenza di moderare le pretese di verità, sostituendo alla categorica formula dell'oltre ogni dubbio 'logico' quella più modesta 
e flessibile dell'oltre ogni 'ragionevole' dubbio: l'aggettivo 'ragionevole' esemplifica bene il fenomeno della sotto-determinazione, mostrando, come in una supplica, l'aspirazione, ma al tempo stesso l'impossibilità di raggiungere una sicurezza assoluta sulla verità della proposizione da provare. Si rinuncia allo standard deduttivo, per ottenere quel grado di persuasività e di fondatezza che è lecito pretendere nel settore empirico: nulla vieta di parlare di 'certezza' anche per le scienze empiriche, purché sia chiaro che non è la medesima certezza delle scienze deduttive.

Sul piano operativo la regola svolge una doppia funzione. Quella più palese è di garantire l'imputato dal rischio di una condanna ingiusta: il giudice può condannare solo quando la colpevolezza abbia trovato piena conferma nelle prove di accusa e nessuna significativa smentita in quelle a favore (altrimenti è doverosa l'assoluzione). Per riprendere una bella immagine di Wittgenstein, 'colpevole al di là di ogni ragionevole dubbio' sta a significare che si è giunti a un punto, indeterminabile a priori, in cui la vanga del dubbio, che deve sempre armare il giudice, ha incontrato lo strato duro della roccia, rappresentata dalle prove, e si è piegata, risultando inaccettabile ogni spiegazione diversa dalla colpevolezza.

Ma, simmetricamente, la clausola vuole anche sottolineare che, se la colpevolezza è suffragata da un solido e coerente quadro probatorio, l'onesto riconoscimento della fallibilità degli accertamenti non deve impedire la condanna. Se il dubbio, astrattamente ipotizzabile, non appare "ragionevole", si può ritenere raggiunta la prova e assumere convenzionalmente il risultato come certo, data l'impossibilità di giustificarlo in termini assoluti e definitivi. È questo l'apporto più significativo dell'attributo 'ragionevole', che ci autorizza a considerare 'provata' una proposizione anche quando, in senso puramente logico, si può dubitare di essa. Suonerebbe provocatorio dire al condannato che la sua colpevolezza è 'sotto-determinata' dalle prove; e, nondimeno, l'aggettivo 'ragionevole', in opposizione a 'logico', esprime proprio un simile concetto.

\section{7. "La prova oltre ogni RagioneVole dubBio" tra regola E PRINCIPIO: I C.D. CASI DIFFICILI.}

Si parla comunemente di regola dell'oltre ogni ragionevole dubbio. Ma si tratta realmente di una regola o piuttosto di un principio? 
Riprendiamo la distinzione dworkiniana tra principi e regole ${ }^{32}$. I principi sono proposizioni normative ad elevato grado di genericità (a fattispecie aperta), applicabili nella forma del "più-o-meno", quindi con la massima espansione o restrizione. Le regole sono proposizioni normative ad elevata specificità (a fattispecie chiusa, secondo il modello "Se A, allora segue B"), applicabili nella forma del "tutto-o-niente", quindi destinate ad essere osservate o trasgredite, senza scelte intermedie.

Esistono, tuttavia, proposizioni che, formalmente strutturate come regole, funzionano sostanzialmente come principi: una di queste è, per l'appunto, rappresentata dalla disposizione che subordina la condanna alla prova della colpevolezza 'oltre ogni ragionevole dubbio'. La forma è quella di una regola, in quanto il giudice, che registri la permanenza di un ragionevole dubbio, è categoricamente tenuto ad assolvere. Ma di fatto la direttiva opera come un principio, perché la verifica sulla ragionevolezza o no del dubbio viene a dipendere da complessi giudizi di valore che stanno al di là della regola e che rendono estremamente elastica la valutazione del giudice.

Espressioni come "ragionevole", "giusto", “equo" presentano ai margini un alone di indeterminatezza, una zona grigia dalla quale possono scaturire opposte soluzioni, tutte legittime. Esistono casi 'facili', connotati da un quadro probatorio univoco, di fronte al quale ogni persona assennata concorderebbe nell'assolvere o nel condannare. Ma, per quanta resistenza vi sia ad ammetterlo, vi sono altri contesti - i c.d. casi 'difficili' - dove il materiale probatorio può giustificare la condanna come l'assoluzione e dove, quindi, la scelta tra l'una e l'altra, pur doverosamente operata dal giudice sul presupposto che quella sia la decisione giusta, è inevitabilmente connotata da una spiccata discrezionalità. Un diverso giudice, altrettanto lucido cognitivamente, potrebbe assumere e motivare l'opposta conclusione nel pieno rispetto della legalità: il dubbio che ad uno appare "ragionevole", può risultare ad altri "irragionevole". Discrezionalità

32 R. Dworkin, I diritti presi sul serio [1977], trad. it., Il Mulino, Bologna, 1982, 93 s. La distinzione è ripresa, nei rapporti tra diritto di difesa ed efficienza del processo, da R. ORLANDI, Garanzie individuali ed esigenze repressive (ragionando intorno al diritto di difesa nei procedimenti di criminalità organizzata), in Studi in memoria di Gian Domenico Pisapia, II, Giuffrè, Milano, 2000, 558 s. 
significa, appunto, scelta tra più opzioni, ciascuna legittima ${ }^{33}$, tanto sul terreno del giudizio storico quanto sul versante del giudizio di diritto; il che, beninteso, non giustifica atteggiamenti di tipo decisionista (se più opzioni sono legittime, tanto vale decidere a caso), dovendo il giudice impegnarsi nella ricerca di ciò che, con Dworkin, potremmo definire l'unica risposta 'giusta' (nella pluralità di quelle legittime).

Nessun giudice, ovviamente, dirà mai ad un condannato che con pari legittimità sarebbe stato assolto, né permetterà che traspaia dalla motivazione della sentenza; ma sarebbe un'ipocrisia negare la presenza di casi 'difficili' che nessun criterio legale di valutazione può eliminare. La linea che divide il colpevole dall'innocente è netta dal punto di vista ontologico; vaga da quello epistemico o conoscitivo, dove a separare l'assoluzione dalla condanna è la 'ragionevolezza” o no del dubbio.'

\section{Il DUBBIO ... SUlLA 'RAGIONEVOLEZzA' DEL DUBBIO.}

Qualcuno obietterà che nei casi ‘difficili', dove appaiono legittime più opzioni, occorre assolvere per il fatto stesso che si profila uno stato d'incertezza, quindi, di 'dubbio'. Ma è facile rispondere che, per quanto si estenda l'area dell'assoluzione, il confine che la divide dalla condanna rimarrà sempre vago. Si può ragionevolmente dubitare se ... il dubbio sia 'ragionevole', e così via, con un regresso all'infinito.

La legge impone l'assoluzione dopo che il giudice abbia ritenuto 'ragionevole' il dubbio sulla colpevolezza. Solo a quel punto interviene la regola di giudizio che, seppure non fosse espressa, discenderebbe già autonomamente dall'onere della prova gravante sull'accusa; nessun giudice condannerebbe un imputato, sulla cui colpevolezza sussista un dubbio ragionevole.

Ma le incertezze che possono determinare un'impasse decisoria si manifestano prima, quando occorre stabilire se il dubbio sia o no ragionevole; è in questa fase che la risposta può apparire problematica, a causa della zona d'ombra che connota il concetto di 'ragionevole'. Si spiegano così le accanite dispute che spesso si registrano in lunghe camere di consiglio.

33 A. BARAK, La discrezionalità del giudice penale [1989], Giuffrè, Milano, 1995, 16 s. 
Qui nessun aiuto, nessuna regola di giudizio arriva dalla legge; a sciogliere il dilemma in un senso o nell'altro, a decidere se il dubbio, astrattamente ipotizzabile, sia o no 'ragionevole', è solo il giudice nella sua pesante responsabilità. $\mathrm{E}$ - sia detto di sfuggita - è proprio nello spazio incerto e nebuloso, aperto da quell'aggettivo, che più intensamente l'accusa e la difesa svolgono la loro attività retorico-argomentativa ${ }^{34}$; maggiore è la discrezionalità del giudice, e più cresce potenzialmente l'influenza delle parti, che viceversa tenderebbe a zero nel sistema di una prova intesa come dimostrazione matematica.

Per questo sbaglia chi s'attende dall'oltre ogni ragionevole dubbio una risposta univoca alle ricorrenti polemiche sulla prova del nesso di causalità in materie come la responsabilità medica, l'esposizione a sostanze nocive per la salute umana, ecc. La formula, come si accennava, nasce proprio dall'impossibilità di quantificare il grado di probabilità necessario per la pronuncia di una condanna; 'dubbio ragionevole' segnala l'assenza di un limite preciso a partire dal quale l'evidenza probatoria denota la colpevolezza. Sarebbe, dunque, paradossale pretendere, con un percorso inverso, di definire quel grado o quel confine sulla base di un attributo dai contorni così vaghi; allo stesso modo in cui nel paradosso del sorite, trovato l'accordo sull'espressione "ragionevole" per definire il numero di granelli idonei a costituire un mucchio, sarebbe poi insensato chiedersi quante unità implichi il numero ragionevole.

Naturalmente la difesa invocherà con insistenza il criterio dell'oltre ogni ragionevole dubbio per ottenere il proscioglimento; ad esempio, affermando che l'accusa non è riuscita a fugare ogni dubbio sulla possibilità che il tumore, contratto dalla vittima esposta ad agenti cancerogeni sul luogo del lavoro, derivi da altre cause o da altri luoghi di esposizione. Argomento risolutivo, se la legge parlasse di colpevolezza 'oltre ogni dubbio', costringendo così il giudice a sistematiche assoluzioni; ma, poiché il dubbio dev'essere 'ragionevole', la scelta decisoria, connotata da ampia discrezionalità, passa attraverso uno standard analogo a quello seguito prima della codificazione del canone in esame. Che il tumore sia

34 R.OrLandi, L'attività argomentativa delle parti nel dibattimento penale, in P. Ferrua-F.Grifantini-G. Illuminati-R. Orlandi, La prova nel dibattimento penale, cit., $457 \mathrm{~s}$. 
stato contratto in un luogo diverso da quello del lavoro, è un dubbio che, pur non avendo nulla di illogico, può nel singolo contesto processuale mostrarsi irragionevole.

\section{L'oltre ogni ragioneVole dubbio e Il teorema di Bayes.}

L'indeterminatezza del concetto di ragionevole dubbio alimenta l'impossibile sogno di trovare una formula esatta sulla cui base si possa ritenere oggettivamente provata una determinata proposizione, a partire ovviamente da quella relativa alla colpevolezza dell'imputato. Al riguardo vi è chi ritiene che un valido aiuto possa essere offerto dal calcolo delle probabilità secondo il teorema di Bayes; e, di recente, il Tribunale di Milano lo ha applicato, pronunciando una sentenza di proscioglimento ${ }^{35}$. Come noto, la formula di Bayes è la seguente:

$$
P(H \mid E)=\frac{P(H) \times P(E \mid H)}{P(E)}
$$

dove con $P(H \mid E)$ si intende la probabilità che ha l'ipotesi di colpevolezza $H$, data l'evidenza probatoria $E$; con $\mathrm{P}(\mathrm{H})$ la probabilità a priori di $H$, ossia indipendente dalle prove; con $P(E \mid H)$ la probabilità che vi sia l'evidenza $E$, assumendo come vera l'ipotesi $H$; $\operatorname{con} P(E)$ la probabilità $a$ priori dell'evidenza $E$, ossia indipendente dalla presenza di $\mathrm{H}$.

La formula può riuscire utile nella parte in cui bene esemplifica e riassume i passi fondamentali da compiere ai fini del giudizio sulla colpevolezza. $P(H)$ corrisponde alla persuasività in sé dell'ipotesi accusatoria,

35 GIP presso il Tribunale di Milano, 18 giugno 2015, in Cass. pen., 2016, 470, p. 3460 con nota di P. Garbolino, Il teorema di Bayes applicato alla prova dattiloscopica trova cittadinanza nel Tribunale di Milano; in precedenza v. J. Della Torre, Il "teorema di Bayes" fa capolino al Tribunale di Milano, in Diritto penale contemporaneo, 21 ottobre 2015. Sulla possibilità di una valutazione quantitativa delle ipotesi e delle prove, v. E. M. Catalano, Logica della prova, statistical evidence e applicazione della teoria delle probabilità nel processo penale, in Diritto penale contemporaneo, 2013, n. 4; A. Mura, Teorema di Bayes e valutazione della prova, in Cass. pen., 2004, 1808 s.; G. Tuzet, Filosofia della prova giuridica, Giappichelli, Torino, 2013, 282 s. 
sganciata da ogni evidenza probatoria. Se la probabilità a priori di $H$ fosse zero, sarebbe pari a zero anche la sua moltiplicazione per l'evidenza probatoria $E$. Assumendo, come ipotesi di scuola, che un pubblico ministero elevasse un'imputazione per un fatto la cui realizzazione appare a priori impossibile - ad esempio, assumendo che l'imputato abbia provocato la morte della vittima trafiggendone l'effige - sarebbe del tutto superfluo procedere ad assunzioni probatorie. Il tema di prova deve rientrare nell'orizzonte del possibile secondo l'epistemologia dominante ${ }^{36}$.

Il fattore $P(E \mid H)$ segnala l'esigenza di accertare quale collegamento sussista tra l'evidenza disponibile e l'ipotesi di colpevolezza; in particolare se appaia verosimile che quell'ipotesi, assunta come vera, possa avere determinato l'evidenza $E$ (parliamo di 'determinare' e non sotto-determinare perché qui il movimento, contrariamente a quello che il giudice dovrà poi operare, è dal passato verso il presente). Infine, il denominatore $P(E)$, relativo alla probabilità a priori di $E$ indica le ipotesi alternative alla colpevolezza, ossia in quale misura l'evidenza probatoria disponibile possa dipendere da cause diverse dall'ipotesi $H$. In breve, la formula di Bayes illustra efficacemente che la prova della colpevolezza oltre ogni ragionevole dubbio implica: a) un fatto possibile secondo l'orizzonte epistemologico dell'epoca; $b$ ) la presenza di prove in stretto collegamento con il fatto e, per converso, l'assenza di prove in significativa contraddizione con esso; c) la non verosimiglianza di ipotesi alternative alla colpevolezza (parliamo di verosimiglianza perché, data la sotto-determinazione, ipotesi alternative sono in linea puramente teorica sempre possibili).

Tuttavia, andare oltre e pretendere di utilizzare il teorema di Bayes per il calcolo delle probabilità di colpevolezza non è, a nostro avviso, possibile per due ragioni ${ }^{37}$. Anzitutto, per la difficoltà di attribuire un preciso valore ai diversi membri della formula, trattandosi di fatti storici e del loro rapporto con le prove del presente: impossibile determinare con precisione la probabilità che l'evidenza disponibile derivi dall'ipotesi accusatoria e in quale misura possa, invece, dipendere da ipotesi alterna-

36 F. Cordero, Procedura penale, IX ed., Giuffrè, Milano, 2012, 572.

37 Sulle difficoltà di adottare una teoria bayesiana della decisione giudiziaria, v. O Mazza, Il ragionevole dubbio nella teoria della decisione, in Criminalia, 2012, n. 1,360 . 
tive. Ma, anche ammesso che si riuscisse ad effettuare quel calcolo, quale sarebbe poi la probabilità necessaria per la condanna, posto che la soglia ideale del $100 \%$ è irraggiungibile nelle inferenze di carattere abduttivo? Non si dimentichi che la scelta del vago aggettivo 'ragionevole' nasce proprio dall'inadeguatezza di qualsiasi determinazione quantitativa in ordine alla probabilità di colpevolezza necessaria per una condanna. È stato saggio troncare l'interminabile discussione con l'elastica regola dell'oltre ogni ragionevole dubbio; a condizione, tuttavia, che nessuno, percorrendo la strada a ritroso, pretenda di stabilire a quale grado di probabilità corrisponda quella regola.

Infine, sebbene non sia possibile qui approfondire il discorso, si può facilmente immaginare che, a meno di abbassare notevolmente la soglia di probabilità per la condanna, la formula di Bayes porterebbe inevitabilmente a sistematiche assoluzioni; e non è un caso che, dove è stata utilizzata, sia avvenuto per avvalorare un proscioglimento a cui il giudice era già palesemente orientato sulla base dell'evidenza probatoria. E persino, nei pochi casi in cui il calcolo delle probabilità portasse a ritenere l'ipotesi di colpevolezza probabile alle irrealistiche soglie di oltre il $95 \%$, si può facilmente immaginare il grido di protesta del condannato: perché proprio io che appartengo a quella piccola percentuale di innocenza? Insomma, l'oltre ogni ragionevole dubbio resta, nella sua preziosa vaghezza, la formula migliore.

\section{FunZIONE PEDAGOGICA DELLA REGOLA DI GIUDIZIO: POVERTÀ DENOTATIVA E RICCHEZZA CONNOTATIVA.}

Come accennato, l'espresso richiamo del codice alla regola dell'oltre ogni ragionevole dubbio nulla aggiunge in termini denotativi a quanto è già implicito nel concetto di 'provare', fuori dall'ambito deduttivo; ma questo non impedisce a quel richiamo di esercitare una notevole influenza sul piano dei significati secondi, di origine pedagogica o retorica. Come è noto, infatti, in una legge quanto più si restringe, sino ad annullarsi, il significato di denotazione, tanto più si espande e si arricchisce quello di connotazione. Vediamo alcuni aspetti.

Anzitutto, vi traspare l'evidente polemica verso una magistratura ritenuta troppo disinvolta nel pronunciare sentenze di condanna; 
e, correlativamente, l'invito per il futuro ad elevare la soglia probatoria necessaria per l'affermazione della colpevolezza. Inoltre, si può scorgere in quella formula una sottolineatura del nesso tra prova e contraddittorio. I dubbi in tanto possono essere ritenuti ragionevoli o irragionevoli in quanto vi sia qualcuno che li prospetti; e nessuno meglio della parte, interessata a confutare la prova, è in grado di farlo.

Beninteso, si tratta del rafforzamento di un'idea che si può già ritenere implicita nel concetto di provare. Popper ha bene illustrato come nella scienza una proposizione sia provata - rectius, provvisoriamente accettata - in quanto abbia resistito ai più severi tentativi di confutazione. A maggior ragione l'assunto deve valere nel processo dove, esauriti i mezzi di impugnazione, le sentenze diventano irrevocabili, quindi inconfutabili; e non è privo di significato che Popper, nell'elaborazione della sua teoria, abbia preso spunto proprio dal contraddittorio che si esercita nel processo davanti alla giuria. Da questo punto di vista qualche perplessità sorge sui provvedimenti cautelari, disposti inaudita altera parte, essendo il contraddittorio differito in sede di impugnazione.

Da notare, infine, un paradossale effetto. Come la maggior parte delle formule povere di significato denotativo, anche questa, una volta immessa nel sistema difficilmente potrà essere estromessa, se non a prezzo di gravi ripercussioni, proprio a causa dei significati di connotazione che veicola. Si può immaginare quali micidiali conseguenze avrebbe, in termini di incoraggiamento a condannare, la caduta della disposizione che ha codificato la regola dell'oltre ogni ragionevole dubbio (art. 533 comma 1 c.p.p.). Nessuno, che non intenda essere additato come forcaiolo, si azzarderà mai a sopprimere o a riformare quella imperitura formula.

\section{Alternative decisorie: termine 'marcato' e termine 'CONSEQUENZIALE'}

La regola della prova oltre ogni ragionevole dubbio segna il livello massimo e, al tempo stesso, minimo affinché nel processo una qualsiasi proposizione possa dirsi provata. Massimo perché la 'sotto-determinazione' della prova induttiva non consente di raggiungere il grado superiore della prova 'indubitabile' nel senso della dimostrazione matematica; minimo 
perché, scendendo sotto quel livello, salta il concetto stesso di provare e si entra nel regno delle illazioni e dei sospetti.

In contrasto con quanto appena affermato, vi è chi ritiene che nel processo la prova dei fatti possa essere raggiunta con diversi standard. Con quello più elevato della regola dell'oltre ogni ragionevole dubbio o con quello inferiore della regola del più probabile che no o con analoghi livelli. La prima regola varrebbe per la prova della colpevolezza ai fini della condanna, la seconda per altri giudizi come quello sulle misure cautelari o sul rinvio a giudizio.

È una prospettiva inaccettabile perché, a scendere sotto il livello della prova oltre ogni ragionevole dubbio, salta il concetto stesso di provare e si entra nel regno delle illazioni e dei sospetti. Al tempo stesso è, però, vero, che ai fini dei provvedimenti cautelari o del rinvio a giudizio non si può pretendere la prova oltre ogni ragionevole dubbio della colpevolezza che si risolverebbe in una condanna anticipata Come uscire dall'impasse? La soluzione sta nel tenere ferma la regola dell'oltre ogni ragionevole dubbio, che è identica per ogni accertamento probatorio, e nell'individuare con esattezza la proposizione da provare che varia invece a seconda del giudizio da emettere.

Più in particolare, in ogni alternativa decisoria si può distinguere: a) un termine 'marcato' che veicola la proposizione o le proposizioni da provare, ossia il tema del giudizio; $b$ ) un termine opposto che si pone come 'consequenziale' all'impossibilità di affermare il termine 'marcato', quindi alla mancata prova (oltre ogni ragionevole dubbio) della proposizione da provare. In altre parole, il termine 'consequenziale' non dispone di un'autonoma proposizione da provare, perché i presupposti per la sua affermazione si riassumono nel fallimento della prova relativa al termine 'marcato'; con la conseguenza che, in situazioni di incertezza sulla prova del termine 'marcato', la scelta deve necessariamente orientarsi verso il termine 'consequenziale' 38 .

Nell'alternativa condanna/proscioglimento il termine marcato è la condanna e la proposizione da provare la colpevolezza. Nell'alternativa archiviazione/rigetto della richiesta di archiviazione il termine marcato è

38 Sul termine 'marcato' e su quello ‘consequenziale', v. P. FerruA, La prova nel processo penale, vol.I, Struttura e procedimento, Giappichelli, Torino, 2017, 107 s. 
l'archiviazione e la proposizione da provare la non idoneità degli elementi raccolti a sostenere l'accusa. Nell'alternativa rinvio a giudizio/sentenza di non luogo a procedere il termine marcato è la sentenza di non luogo a procedere e la proposizione da provare la formula che sta a base della sentenza. Nei provvedimenti cautelari il termine marcato è l'applicazione della misura cautelare e le proposizioni da provare sono, da un lato, il fumus boni iuris, ossia la probabile colpevolezza, dall'altro, il periculum libertatis, ossia il pericolo di fuga o di inquinamento delle prove o della commissione di gravi delitti.

La distinzione tra i due termini si riflette sugli obblighi motivazionali. La decisione del giudice di affermare il termine 'marcato' va suffragata da una motivazione che documenti la prova della proposizione da provare. Quanto al termine 'consequenziale', la motivazione dipende da una scelta discrezionale del legislatore; e, quando sia prevista, può risolversi nell'enunciazione delle ragioni per cui non si è affermato il termine 'marcato'.

\section{Onere della prova.}

Di ‘onere della prova' nel processo penale non si può parlare in senso proprio: l'espressione, infatti, implicherebbe la validità della prova solo se prodotta dalla parte interessata ad affermare la proposizione da provare. Viceversa, secondo il codice vigente, la prova è validamente assunta, chiunque l'abbia prodotta (accusa, difesa o giudice).

Volendo, si può parlare di onere della prova come di 'rischio per la mancata prova'39: 'onerata' della prova in rapporto ad una determinata proposizione fattuale, che concretizza la fattispecie legislativa, è la parte alla quale riesce sfavorevole la decisione in caso di mancata prova della proposizione stessa. Fondamentale è la distinzione tra fatti costitutivi e fatti impeditivi. Vediamo alcuni esempi:

(a) 'è punito con la pena $x$ chi commette il fatto $y$ '

(b) 'è punito con la pena $x$ chi ha commesso il fatto $y$ in assenza del fatto $z$ ' (o formule equivalenti, come: 'quando non sussista $z$ ', 'sempre che non sussista $\left.z^{\prime}\right)$

39 Così, vigente il codice abrogato, F. Cordero, Procedura penale, IX ed., Giuffrè, Milano, 1987, 1008. 
(c) 'è punito con la pena $x$ chi commette il fatto $y$ '; 'è esente da pena chi ha commesso il fatto $y$ in presenza di $z$ ';

(d) 'è punito con la pena $x$ chi commette il fatto $y$, a meno che sussista $z$ '

In (a) fatto costitutivo della colpevolezza è $y$; la sua mancata o insufficiente prova implica la pronuncia dell'assoluzione. Altrettanto dicasi per (b), dove y e $z$ sono i fatti costitutivi della colpevolezza, positivo l'uno, negativo l'altro. La mancata o insufficiente prova del fatto $y$ o dell'assenza di $z$, implica la pronuncia dell'assoluzione. In entrambi i casi il rischio per la mancata prova del fatto positivo o del fatto negativo cade sull'accusa che ha esercitato l'azione penale; la situazione d'incertezza gioca a favore dell'imputato.

In (c) fatto costitutivo della colpevolezza è $y$, mentre fatto impeditivo è $z$. La mancata o insufficiente prova di $z$, quando sia provato $y$, determina la condanna. Qui il rischio per la mancata prova di z cade sulla difesa dell'imputato che si vede condannato: la situazione d'incertezza gioca a favore del pubblico ministero. Ad identica conclusione si deve giungere per (d). Dietro l'apparente unicità, in realtà vi sono due disposizioni: la prima, in forza della quale l'imputato è punito quando abbia commesso il fatto $y$ (onerato della prova è il pubblico ministero); la seconda, in forza della quale l'imputato va esente da pena quando sussista $z$ (onerato della prova è l'imputato). In genere, nel processo penale il rischio per la mancata prova grava sul pubblico ministero, ma non mancano casi in cui grava sull'imputato: ad esempio, nella fattispecie contemplata dall'art. 526 comma 1-bis c.p.p., è onere della difesa provare che il dichiarante si sia sempre sottratto per 'libera scelta' al contraddittorio.

Si conferma così quanto osservato in precedenza. Salvo diversa disciplina, a scandire la ripartizione degli oneri probatori è la struttura della fattispecie (sostanziale o processuale) da cui deriva la proposizione da provare. La previsione legislativa di specifiche regole di giudizio nel processo ha senso nella stretta misura in cui si intenda derogare a quanto logicamente discende dal tema della prova. In genere la 'correzione' è dettata a vantaggio dell'imputato (ad esempio, sul terreno delle cause di giustificazione, dove è prescritta l'assoluzione anche in caso di dubbio sulla loro esistenza: art. 530 comma 3, parte II c.p.p.); ma, talvolta, anche a suo danno (ad esempio, sul terreno delle 
misure cautelari l'art. 275 comma 3 c.p.p., relativamente a certi delitti, converte il fatto costitutivo della sussistenza dei pericula libertatis nel fatto impeditivo della loro assenza.

\section{L'Oltre ogNI RAGIONEVOLE DUBBIO E IL RISCHIO DELL'ABUSO NEI PROVVEDIMENTI CAUTELARI}

Qualcuno si chiederà se l'applicazione dell'oltre ogni ragionevole dubbio ad ogni contesto probatorio non finisca per risolversi in un gioco di parole. Che differenza c'è tra dire che nei provvedimenti cautelari si prova (oltre ogni ragionevole dubbio) la probabile colpevolezza e dire che in essi si dà una prova incompleta, un principio di prova sulla colpevolezza? Alla lunga il risultato non è molto dissimile, o meglio la differenza si riduce a questo: in un caso si tiene ferma la regola dell'oltre ogni ragionevole dubbio, ma si formula in termini probabilistici la proposizione da provare (la probabile colpevolezza); nell'altro, si parla tout court di colpevolezza, ma si adotta un concetto di 'provare' decisamente indebolito, che convive con la presenza di ragionevoli dubbi. Ma che a variare sia la proposizione da provare o l'atto del provare, sarebbe una questione puramente teorica che non incide sulla sostanziale identità del risultato.

Ora è vero che, per quanto attiene al fumus dei provvedimenti cautelari, l'esito dell'una o dell'altra prospettiva non è molto diverso, almeno all'apparenza. Lo è, tuttavia, sul terreno delle esigenze cautelari, dove la proposizione da provare è individuata dalla legge come situazione di 'pericolo': qui indebolire il concetto di 'provare' sino a ritenere sufficiente un principio di prova o una prova incompleta sulla sussistenza del 'pericolo' equivale a vanificare l'accertamento richiesto dalla legge, perché aggiunge all'inevitabile vaghezza dell'espressione 'pericolo' anche l'evanescenza dell'atto di provare.

Ma, più in generale, l'estromissione della regola dell'oltre ogni ragionevole dubbio dal concetto di 'provare' nasconde un'operazione culturale fortemente regressiva. Di fatto conduce a liquidare la necessità stessa di una prova nelle sedi diverse dalla decisione finale sulla colpevolezza; tendenza della quale si vede un saggio nell'indirizzo 
giurisprudenziale e dottrinale che, contrapponendo indizi a prove, nega la necessità delle seconde nei provvedimenti cautelari ${ }^{40}$. Sostenere che nelle decisioni de libertate si possa prescindere da prove o che la valutazione di queste non debba rispettare la regola dell'oltre ogni ragionevole dubbio, indebolisce gravemente le garanzie e la funzione cognitiva del processo, alimentando il rischio di decisionismo, ossia di una prevalenza della componente imperativa rispetto a quella conoscitiva nei provvedimenti del giudice. Qualsiasi accertamento che non sia solidamente ancorato a prove ha molte possibilità di risolversi in un atto di arbitrio.

Il rischio dell'abuso è tanto più presente nei provvedimenti di coercizione personale per una ragione 'psicologica', legata al diverso atteggiamento del magistrato a seconda che sia chiamato a decidere sulla colpevolezza o sulle misure cautelari. Mentre le possibilità che un evento futuro contraddica la condanna o l'assoluzione sono pari, potendo le prove sopravvenire a favore come a discarico, non altrettanto accade sul terreno dei provvedimenti cautelari. Qui la negazione del periculum libertatis può ricevere una plateale smentita nella successiva commissione di un reato (o nell'inquinamento delle prove o nella fuga dell'imputato), esponendo a pesanti critiche il magistrato che abbia rifiutato il provvedimento cautelare. Per converso, difficilmente si potrà documentare, in termini controfattuali, l'assenza del pericolo sulla cui base è stata emessa la misura; la neutralizzazione del pericolo neutralizza anche la possibilità della sua smentita e nessuno riuscirà a provare che il contegno dell'imputato in libertà sarebbe stato innocuo. Di qui una sorta di principio di 'precauzione' che di fatto tende a prevalere sul favor libertatis, amplificando la rilevanza dei pericoli che giustificano la custodia cautelare: nel dubbio sulla loro sussistenza, il magistrato propende al pessimismo, si sente più 'rassicurato' dall'affermazione che dalla negazione del pericolo, consapevole che solo quest'ultima scelta può essere retrospettivamente sconfessata.

40 V. la sentenza costituzionale n. 131 del 1996 dove si afferma che il giudizio prognostico ex art. 273 c.p.p. è condotto «non su prove, ma su indizi»; nello stesso senso, per la contrapposizione tra indizi e prove, Corte cost. sentenza n. 432 del 1995, nonché Cass., Sez. un., 30 ottobre 2002, Vottari, in Cass. pen., 2003, 396, nt. 112 . 


\section{Presunzione di innocenza e prova oltre ogni RAGIONEVOLE DUBBIO.}

È opinione corrente che sussista uno stretto collegamento tra la regola dell'oltre ogni ragionevole dubbio e i principi contenuti nell'art. 27 comma 2 Cost. («L'imputato non è considerato colpevole sino alla condanna definitiva») e nell'art. 6 comma 2 CEDU («Ogni persona accusata di un reato è presunta innocente sino a quando la sua colpevolezza non sia stata legalmente accertata») ${ }^{41}$.

Come si accennava, che il processo abbia come tema la colpevolezza e che questa sia da provare oltre ogni ragionevole dubbio, deriva dalla struttura della fattispecie penale e dal concetto di 'provare'; e sin qui nulla muterebbe, anche in assenza dell'art. 27 comma 2 Cost. e dell'art. 6 comma 2 CEDU. Essendo la fattispecie penale imperniata sulla punibilità di chi abbia commesso un determinato fatto, la pena in tanto può essere applicata in quanto sia provata sino all'ultima molecola la commissione del fatto, salve le deroghe previste dal codice di rito: ad esempio, in ordine al patteggiamento (artt. 444-448 c.p.p.) o alla messa alla prova (artt. 464 bis-novies c.p.p.).

Non per questo, tuttavia, quelle disposizioni devono ritenersi superflue. A parte la regola di trattamento che ne discende, il principio della presunzione di innocenza ha l'importante effetto di imporre al legislatore due obblighi. In sede sostanziale, l'impegno a non costruire fattispecie basate su sospetti o illazioni, dove di fatto la colpevolezza verrebbe a consistere nell'impossibilità di giustificare l'innocenza; e, in sede processuale, a non alterare in malam partem le regole sull'onere della prova, ad esempio, istituendo presunzioni sui fatti costitutivi della colpevolezza o sui presupposti delle misure cautelari. Giustamente la Corte costituzionale ha censurato, fra le disposizioni del primo tipo, l'art. 12-quinquies, secondo comma, del decreto-legge 8 giugno 1992, n. $306^{42}$; e, fra le disposizioni del secondo tipo, l'art. 275 comma 3 c.p.p.

41 C. Santoriello, Il vizio di motivazione, cit., $52 \mathrm{~s}$.

42 La Corte costituzionale, con sentenza n. 48 del 1994, ha dichiarato illegittimo, per contrasto con l'art. 27 comma 2 Cost., l'art. 12-quinquies, secondo comma, del decreto-legge 8 giugno 1992, n. 306 (Modifiche urgenti al nuovo codice di procedura penale e provvedimenti di contrasto alla criminalità 
nella parte in cui, per determinati reati, imponeva la custodia cautelare in carcere, senza far salva «l'ipotesi in cui siano acquisiti elementi specifici, in relazione al caso concreto, dai quali risulti che le esigenze cautelari possono essere soddisfatte con altre misure ${ }^{43}$.

Restano, invece, possibili, ed anzi in qualche modo incoraggiate dal principio della presunzione di innocenza, deroghe all'onere della prova operanti in bonam partem, come quella sulla prova delle cause di giustificazione (art. 530 comma 3 c.p.p.).

\section{Bibliografia}

CAPRIOLI, Francesco. Verità e giustificazione nel processo penale. Riv. it. dir. proc. pen., 2013, $623 \mathrm{~s}$.

CAPRIOLI, Francesco. Scientific evidence e logiche del probabile nel processo per il “delitto di Cogne". Cass. pen., 2009, 1867 s.

CARNELUTTI, Francesco. La prova civile (1915). Milano: Giuffrè, 1992.

CATALANO, Elena Maria. Ragionevole dubbio e logica della decisione. Milano: Giuffrè, 2016.

CORDERO, Franco. Procedura penale, 9 ed. Milano: Giuffrè, 2012.

DANIELE, Marcello. Regole di esclusione e regole di valutazione della prova. Torino: Giappichelli, 2009.

FERRUA, Paolo. Il giudizio penale: fatto e valore giuridico. In: FERRUA; GRIFANTINI; ILLUMINATI e ORLANDI, La prova nel dibattimento penale, 4 ed., Torino, $2010,317 \mathrm{~s}$.

FERRUA, Paolo. La prova nel processo penale, I, Struttura e procedimento. 2 ed. Torino: Giappichelli, 2015.

mafiosa), convertito, con modificazioni, dalla legge 7 agosto 1992, n.356, come modificato dall'art. 1 del decreto-legge 17 settembre 1993, n.369 (Disposizioni urgenti in tema di possesso ingiustificato di valori e di delitti contro la pubblica amministrazione), convertito, con modificazioni, dalla legge 15 novembre 1993, n. 461.

${ }^{43}$ FERRUA, Paolo. La prova nel processo penale. Vol. I, Struttura e procedimento. $2^{\circ}$ ed. Torino: Giappichelli, 2017. p. 113 ss. 
ILLUMINATI, Giulio. La presunzione d'innocenza dell'imputato. Bologna: Zanichelli, 1979.

MAZZA, Oliviero. Il ragionevole dubbio nella teoria della decisione. Criminalia, 2012, 357 s.

PAULESU, Pier Paolo. La presunzione di non colpevolezza dell'imputato. Torino: Giappichelli, 2009.

SANTORIELLO, Ciro. La prova penale e la sua valutazione. Roma: Aracne, 2012.

TONINI, Paolo; CONTI, Carlotta. Il diritto delle prove penali. 2 ed. Milano: Giuffrè, 2014.

TUZET, Giovanni. Filosofia della prova giuridica. Torino: Giappichelli, 2013.

UBERTIS, Giulio. La prova penale. Profili giuridici ed epistemologici. Torino: Utet, 1995.

\section{Informações adicionais e declarações dos autores (integridade científica)}

Declaração de conflito de interesses (conflict of interest declaration): o autor confirma que não há conflitos de interesse na realização das pesquisas expostas e na redação deste artigo.

Declaração de coautoria e especificação das contribuições (declaration of authorship): todas e somente as pessoas que atendem os requisitos de autoria deste artigo estão listadas como autores; o autor se responsabiliza integralmente por este trabalho em sua totalidade.

Declaração de ineditismo e originalidade (declaration of originality): este artigo é uma versão parcial e revisada do trabalho originalmente publicado em: FERRUA, Paolo. La prova nel processo penale. Vol. I, Struttura e procedimento. $2^{\circ}$ ed. Torino: Giappichelli, 2017; o autor assegura que não há plágio de terceiros. 
Dados do processo editorial

(http://www.ibraspp.com.br/revista/index.php/RBDPP/about/editorialPolicies)

- Recebido em: 13/12/2017

Equipe editorial envolvida

- Retorno rodada de correções: 31/01/2018

- Editor-chefe: 1 (VGV)

- Autor convidado

- Editor-associado: 1 (GHB)

http://www.ibraspp.com.br/revista/index.php/

RBDPP/about/editorialPolicies - custom-1

- Editora-assistente: 1 (BC)

\section{COMO CITAR ESTE ARTIGO:}

FERRUA, Paolo. La prova nel processo penale. Revista Brasileira de Direito Processual Penal, Porto Alegre, vol. 4, n. 1, p. 81-128, jan./mai. 2018.

https://doi.org/10.22197/rbdpp.v4i1.130

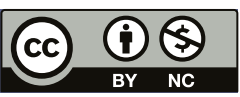

Esta obra está licenciada com uma Licença Creative Commons Atribuição-NãoComercial 4.0 Internacional. 